\title{
Inhibition of ROCK signaling pathway accelerates enteric neural crest cell-based therapy after transplantation in a rat hypoganglionic model
}

\author{
Yuying Zhao ${ }^{1,2} \mid$ Xin Ge ${ }^{1}$ | Hui Yu ${ }^{1}$ | Laura E. Kuil ${ }^{2}$ | Maria M. Alves ${ }^{2}$ | \\ Donghao Tian $^{1}$ | Qiang Huang ${ }^{1}$ | Xinlin Chen ${ }^{3}$ | Robert M. W. Hofstra ${ }^{2}$ | Ya Gao ${ }^{1}$
}

${ }^{1}$ Department of Pediatric Surgery, The Second Affiliated Hospital of Xi'an Jiaotong University, Xi'an, Shaanxi, China

${ }^{2}$ Department of Clinical Genetics, Erasmus University Medical Center, Rotterdam, The Netherlands

${ }^{3}$ Institute of Neurobiology, Xi'an Jiaotong University Health Science Center, Xi'an, Shaanxi, China

\section{Correspondence}

Ya Gao, Department of Pediatric Surgery, The Second Affiliated Hospital of Xi'an Jiaotong University, No 157, Xi Wu Road, Xi'an 710004, Shaanxi, China.

Email: ygao@xjtu.edu.cn

Funding information

National Natural Science Foundation of China, Grant/Award Number: 81770513 and 81741096

\begin{abstract}
Background: Hirschsprung's disease (HSCR) is a congenital gastrointestinal disorder, characterized by enteric ganglia absence in part or entire of the colon, due to abnormal colonization and migration of enteric neural crest cells (ENCCs) during development. Currently, besides surgery which is the main therapy for HSCR, the potential of stem cell-based transplantation was investigated as an alternative option. Although promising, it has limitations, including poor survival, differentiation, and migration of the grafted cells. We hypothesized that modulation of extracellular factors during transplantation could promote ENCCs proliferation and migration, leading to increased transplantation efficiency. Considering that the RhoA/ROCK pathway is highly involved in cytoskeletal dynamics and neurite growth, our study explored the effect of inhibition of this pathway to improve the success of ENCCs transplantation. Methods: Enteric neural crest cells were isolated from rat embryos and labeled with a GFP-tag. Cell viability, apoptosis, differentiation, and migration assays were performed with and without RhoA/ROCK inhibition. Labeled ENCCs were transplanted into the muscle layer of an induced hypoganglionic rat model followed by intraperitoneal injections of ROCK inhibitor. The transplanted segments were collected 3 weeks after for histological analysis.

Key Results: Our results showed that inhibition of ROCK increased viable cell number, differentiation, and migration of ENCCs in vitro. Moreover, transplantation of labeled ENCCs into the hypoganglionic model showed enhanced distribution of grafted ENCCs, upon treatment with ROCK inhibitor.

Conclusions and Inferences: ROCK inhibitors influence ENCCs growth and migration in vitro and in vivo, and should be considered to improve the efficiency of ENCCs transplantation.
\end{abstract}

KEYWORDS

ENCCs, enteric nervous system, hirschsprung's disease, RhoA/ROCK pathway, transplantation 


\section{1 | INTRODUCTION}

Hirschsprung's disease (HSCR) is a congenital gut motility disorder, characterized by the absence of enteric ganglia along different intestinal lengths. It results from the failure of enteric neural crest cells (ENCCs) to colonize the gut during fetal development. ${ }^{1}$ Genetic studies have identified rare coding and non-coding variants in several genes as the leading cause of HSCR, but they only explain 30\% of the cases. ${ }^{2}$ HSCR has an incidence of 1:5000 live births and is characterized by constipation, abdominal distension, and megacolon. At present, surgical removal of the aganglionic segment is the main treatment for HSCR. ${ }^{3}$ However, in some cases, surgery cannot relieve all symptoms and can lead to secondary complications, such as enterocolitis. Therefore, there is a need for novel treatment options for HSCR.

Over the last 10-15 years, stem cell-based therapy to replace the missing enteric nervous system (ENS) in HSCR has been investigated. Transplanting ENCCs into the aganglionic bowel could lead to an improvement of gut motility in animal models. ${ }^{4-7}$ However, ENCC transplantation has limitations, namely poor survival, differentiation, and migration of the grafted cells. ${ }^{8}$ It was speculated that these limitations could be attributed to the overall condition of the grafted cells on the one hand and the condition of the environment in which cells were transplanted on the other hand. In the latter, collagen overabundance, ${ }^{9,10}$ inflammation cascade, ${ }^{11}$ and growth cytokine deficiency seem to play a role. Therefore, it is reasonable to assume that the modulation of extracellular factors during transplantation could promote engraftment and ultimately lead to an increase in the transplantation efficiency.

The RhoA/ROCK pathway consists of RhoA and downstream target homologous isomers, ROCK1, and ROCK $2{ }^{12}$ Regulation of this pathway is essential for cytoskeletal dynamics and cell motility. ${ }^{13}$ ROCK activation can inhibit axonal growth and neural regeneration in the central nervous system. ${ }^{14,15}$ Based on these findings, inhibition of the RhoA/ROCK pathway is considered a rational treatment approach for neurological disorders, with several studies demonstrating remarkable recovery effects using inhibitors like Y-27632, Fasudil, and BA-210. ${ }^{16,17}$ Moreover, the migration and axon extension of enteric neurons was found increased upon inhibition of the ROCK pathway by Y-27632 exposure, ${ }^{18}$ possibly by permitting RAC1 activation. ${ }^{19}$ Apart from the nervous system, the ROCK pathway is also involved in the regulation of intestinal smooth muscles contraction. ${ }^{20}$ Hyper-activation of Rho-kinase has also been reported in the aganglionic segment of endothelin-B receptor gene-deficient rats $\left(E d n r b^{-/-}\right)$, a known HSCR model. ${ }^{21}$ Therefore, the precise function of the RhoA/ROCK pathway on the ENS requires further research, but it seems reasonable that inhibition of the RhoA/ROCK pathway could be one of the factors that positively affects the ENCCs based therapy after transplantation.

In this study, we investigated the role of the RhoA/ROCK pathway in both ENCC culture and transplantation into the hypoganglionic segments of rat models. We hypothesized that inhibition of this pathway could improve transplantation efficiency by promoting the

\section{Key points}

- Enteric neural crest cells (ENCCs) based therapy for Hirschsprung's disease (HSCR) has obtained promising progress, but there are major limitations including poor survival, differentiation, and migration ability of transplanted ENCCs.

- Modulation of environmental factors during transplantation could promote transplantation efficiency, functioning as a potential optimizing target.

- Results in the present work suggest that inhibition of the ROCK pathway improved the growth, differentiation, and migration of ENCCs in vitro, and enhanced distribution of grafted ENCCs after transplantation in vivo.

- This study highlights the impact of the RhoA/ROCK pathway on enteric neural cells and emphasizes the importance of the extracellular environment in Hirschsprung's disease.

growth, differentiation, and migration of ENCCs, thereby leading to a more successful transplantation outcome.

\section{MATERIALS AND METHODS}

\section{1 | Animal preparation}

One hundred adult Sprague-Dawley (SD) rats (both sexes) with a bodyweight of $200 \pm 50 \mathrm{~g}$ and twenty SD pregnant female rats were obtained from the Experimental Animal Center of Xi'an Jiaotong University, Health Science Center. They were used to establish the hypoganglionic model (see 2.8) or to isolate the ENCCs primary cultures (see 2.2), respectively. All rats were socially housed and fed with standard diet and water. The bodyweight was measured every other day. All animals were handled in accordance with the guidelines provided by the ethics committee and the National Health Institute regarding the care and use of laboratory animals. The experiment additionally adhered to ARRIVE guideline standards.

\subsection{ENCCs culture}

Pregnant SD rats carrying 14-day-old embryos were sacrificed by administering an overdose of anesthesia (7\% chloral hydrate). Embryos were delivered via cesarean section. The intestinal tract of each embryo was isolated and put on ice. Mesentery and vessels were carefully removed under a stereomicroscope. The intestines were cut into pieces of $1 \mathrm{~mm}$ and dissociated with $0.25 \% \mathrm{w} / \mathrm{v}$ collagenase IV (Gibco, 17104019) and 0.25\% w/v dispase II (Sigma, 42613-33-2), for 40 minutes, at $37^{\circ} \mathrm{C}$. During incubation, the sample was vortexed every 5 minutes. Subsequently, the cell suspension was 
filtered through a $100 \mu \mathrm{m}$ mesh filter and centrifuged at $800 \mathrm{rpm}$ for 4 minutes. Cells were resuspended and plated at a density of $2 \times 10^{5}$ cells $/ \mathrm{mL}$ in complete culture medium, which was DMEM F12 (1:1) (Hyclone, SH30023.01), supplemented with 1.0\% N2 supplement (Gibco, A1370701), 1.0\% B-27 supplement (Gibco, 12587010), $5 \mathrm{ng} / \mathrm{mL}$ recombinant basic fibroblast growth factor (bFGF) (Gibco, PHG0021), $5 \mathrm{ng} / \mathrm{mL}$ recombinant epidermal growth factor (EGF) (Gibco, PHG0311), $2 \mathrm{mmol} / \mathrm{L}$ L-glutamine, and $100 \mathrm{U} / \mathrm{mL}$ penicillinstreptomycin (Gibco, 15140148). Cells were cultured at $37^{\circ} \mathrm{C}$, in $5 \%$ $\mathrm{CO}_{2}$, for 7 days to form neurospheres.

To split the ENCCs, neurospheres were harvested by centrifugation and dissociated into smaller neurospheres using $0.25 \% \mathrm{w} / \mathrm{v}$ collagenase IV and dispase II, at $37^{\circ} \mathrm{C}$ for 5 minutes. Cell clusters were re-cultured in complete culture medium. To identify ENCCs markers, neurospheres were harvested, fixed, and dropped on coverslips for immunofluorescence staining (see 2.10).

\subsection{Viable cell counting assay}

Single ENCCs obtained by trypsinization of neurospheres were seeded at a density of 4000 cells/well in 96-well plates in complete culture medium (see 2.2). Forty-eight hours later, cells were treated with different RhoA/ROCK inhibitors: CCG-1423 (APExBIO, B4897), Y-27632 dihydrochloride (APExBIO, A3008), SR-3677 (APExBIO, B1294), or with PBS. For each inhibitor, the following concentrations were used: 0.01, 0.1, 1, 10, $100 \mu \mathrm{mol} / \mathrm{L}$. After 72 hours of treatment, cells in 24 -well plates were imaged and counted. Cells in 96-well plates were used to assess cell viability using the Cell Counting Kit-8 (7-Sea Biotech, C008-3), according to the manufacturer's instructions. In brief, the CCK-8 kit uses WST-8 tetrazolium sodium salt, which produces a water-soluble formazan dye upon bio-reduction by cellular dehydrogenases. The amount of formazan produced is directly proportional to the number of living cells; therefore, the absorbance (OD optical density) at $450 \mathrm{~nm}$ indicates viable cell numbers, which was measured 4 hours later after addition of WST- 8 .

\subsection{Differentiation of ENCCs under treatment with inhibitors}

Single ENCCs obtained by trypsinization of neurospheres were seeded at a density of $4 \times 10^{5}$ cells/well in 6-well plates, and $1 \times 10^{5}$ cells/well on coverslips in 24 -well plates, in the complete culture medium (see 2.2). After 48 hours, $2 \%$ fetal calf serum (FCS) (Gibco, A3161001C) was added to each well to induce differentiation. Thereafter, cells were treated with different inhibitors: $10 \mu \mathrm{mol} / \mathrm{L}$ CCG-1423, $10 \mu \mathrm{mol} / \mathrm{L}$ Y-27632 dihydrochloride, and $1 \mu \mathrm{mol} / \mathrm{L} \mathrm{SR}-3677$ for 72 hours. PBS was used as control. After treatment, cells in the 6-well plates were lysed for Western blotting, and cells on coverslips were fixed and immunostained (see 2.10).

\section{5 | Trans-well migration assays}

ENCC migration was assessed using Millicell Hanging Cell Culture Inserts (Millipore) in 24-well plates. A total of $200 \mu \mathrm{L}$ of single ENCC suspension trypsinized from neurospheres were plated at a density of $5 \times 10^{4}$ cells $/ \mathrm{mL}$ in complete culture medium (see 2.2 ) in the top chamber. In addition, $600 \mu \mathrm{L}$ of complete culture medium supplemented with $2.0 \%$ FCS was added to the bottom chamber for chemo-attraction. Thereafter, either $10 \mu \mathrm{mol} / \mathrm{L}$ CCG-1423, $10 \mu \mathrm{mol} / \mathrm{L} \mathrm{Y-27632,} 1 \mu \mathrm{mol} / \mathrm{L} \mathrm{SR}-3677$, or PBS (used as control) were added to both the top and bottom chambers. After 72 hours of incubation, cells were fixed on the membrane with methanol and stained with gentian violet. The number of cells that passed through the membrane was counted to assess migration.

\section{6 | Cell apoptosis assay}

Single ENCCs trypsinized from neurospheres were plated in 6-well plates at $4 \times 10^{5}$ cells/well in complete medium (see2.2). 48 hours later, cells were treated with $10 \mu \mathrm{mol} / \mathrm{L}$ of CCG-1423, $10 \mu \mathrm{mol} / \mathrm{L}$ of $\mathrm{Y}-27632,1 \mu \mathrm{mol} / \mathrm{L}$ of SR-3677, or PBS. After 72 hours of treatment, cells were collected and apoptosis was determined using the Annexin V: PE Apoptosis Detection Kit I (BD, 559763) according to the manufacturer's instructions.

\section{7 | ENCC labeling with a fluorescent tag}

Lentivirus vector HBLV-GFP-PURO was obtained from HANBIO (Han Biotech, 04021724, Shanghai). Transduction was performed according to the manufacturer's protocol. In brief, small ENCC colonies obtained from gentle dissociation were seeded in 6-well plates at a density of $4 \times 10^{5}$ cells/well. Transduction was performed on day two with different multiplicity of infection (MOI): $5 \times, 25 \times, 125 \times$, or $250 \times$ lentivirus, along with $6 \mu \mathrm{g} / \mathrm{mL}$ polybrene (Han Biotech, HBPB-500). Plates were centrifuged at $37^{\circ} \mathrm{C}, 500 \times g$ for 30 minutes, and then returned to the incubator. The medium was changed on day 4 , and the cells were analyzed on day 6 via fluorescent imaging and FACS. The FACS gating strategy was delimited to distinguish GFP ${ }^{+}$ cells according to negative control population.

\subsection{Establishment of the rat hypoganglionic model}

All surgical procedures used in this study had been previously described. ${ }^{22}$ In summary, the procedure is as follows. Preoperative fasting was performed for 8 hours. Rats were operated with a midline incision under anesthesia ( $7 \%$ chloral hydrate). A soft tube, with a diameter of $2 \mathrm{~mm}$, was inserted into the anus as an indicator of the length. The descending colon segment located $5 \mathrm{~cm}$ away from anus, was wrapped in 1-cm-wide filter paper soaked thoroughly with $0.1 \%$ benzalkonium chloride (BAC, Tokyo Chemical Industry, 


\begin{tabular}{|c|c|c|c|c|c|}
\hline Antibody & Brand & Cat\# & RRID & Application & dilution \\
\hline anti-p75 NTR & Abcam & ab8874 & AB_306827 & $\mathrm{IF}$ & $1: 200$ \\
\hline anti-Nestin & Abcam & ab11306 & AB_1640723 & IF & $1: 200$ \\
\hline $\begin{array}{l}\text { anti- } \beta-\text {-III Tubulin } \\
\text { (Tuj1) }\end{array}$ & Genetex & GTX631836 & AB_2814952 & IF & $1: 200$ \\
\hline anti-GFAP & Abcam & ab33922 & AB_732571 & IF & $1: 200$ \\
\hline anti-NeuN & Abcam & ab177487 & AB_2532109 & IF & $1: 200$ \\
\hline anti-GFP & Abcam & ab6556 & AB_305564 & $\mathrm{IF}$ & $1: 500$ \\
\hline $\begin{array}{l}\text { Goat anti-Rabbit IgG } \\
\text { H\&L Alexa Fluor } \\
488\end{array}$ & Abcam & ab150077 & AB_2630356 & $\mathrm{IF}$ & $1: 400$ \\
\hline $\begin{array}{l}\text { Goat anti-Mouse IgG } \\
\text { H\&L Cy3 }\end{array}$ & Abcam & ab97035 & AB_10680176 & IF & $1: 400$ \\
\hline anti-PGP9.5 & Genetex & GTX109637 & AB_1952497 & $\mathrm{IHC}$ & $1: 200$ \\
\hline $\begin{array}{l}\text { anti- } \beta-\text { III Tubulin } \\
\text { (Tuj1) }\end{array}$ & Genetex & GTX631836 & AB_2814952 & $\mathrm{IHC}$ & $1: 200$ \\
\hline anti-GFAP & Abcam & ab33922 & AB_732571 & WB & $1: 1000$ \\
\hline $\begin{array}{l}\text { anti- } \beta-\text { III Tubulin } \\
\text { (Tuj1) }\end{array}$ & Genetex & GTX631836 & AB_2814952 & WB & $1: 1000$ \\
\hline anti- $\beta$-actin & Abcam & $a b 8226$ & AB_306371 & WB & $1: 2000$ \\
\hline $\begin{array}{l}\text { EasyBlot anti-Mouse } \\
\text { IgG (HRP) }\end{array}$ & Genetex & GTX221667-01 & AB_10728926 & WB & $1: 1000$ \\
\hline $\begin{array}{l}\text { EasyBlot anti-Rabbit } \\
\text { IgG (HRP) }\end{array}$ & Genetex & GTX221666-01 & AB_10620421 & WB & $1: 2000$ \\
\hline
\end{tabular}

TABLE 1 Antibodies List of antibodies used in this experiment

GFAP, glial fibrillary acidic protein.

NeuN, neuron nuclear protein.

P75NTR, P75 neurotrophin receptor.

PGP9.5, protein gene product 9.5 .

Tuj1, $\beta$-III-tubulin.

63449-41-2), and remained wrapped for 30 minutes. A medical gauze was used to isolate the treated colon from the abdominal cavity. After removing the filter paper, the target segment was washed three times thoroughly with saline and returned to its original position. Histological evaluation of treated segments was performed at 2,4 , and 6 weeks after surgery, by immunohistochemistry of betaIII-tubulin (Tuj1) and protein gene product 9.5 (PGP9.5), in order to determine ENS status.

\section{9 | ENCCs transplantation}

Three weeks after establishment of the hypoganglionic model, transplantation was performed under the same fasting and anesthesia conditions. Three different groups having 20 rats each were divided as follows: ENCC-only group, ENCC + Y-27632 group, and the control group. For the first two groups, $5 \times 10^{6}$ cells $/ \mathrm{mL}$ GFPlabeled ENCCs were transplanted into the hypoganglionic segments by microinjection of $20 \mu \mathrm{L}$ cell suspension for each injection into the muscle layers at the central surface at 0.3.6.9 clockwise position (see Figure 6B). The control group was injected with PBS under the same conditions.
Continued treatment with the inhibitor started 1 day after surgery by intraperitoneal injection of $0.07 \mathrm{mg} / \mathrm{kg} \mathrm{Y}-27632$ into ENCC + Y-27632 group, every alternate day. Normal saline was injected into the ENCC-only group and control group using the same protocol. Three weeks after the transplantation, the rats were sacrificed and colon segments were isolated, fixed, and paraffin-embedded in a longitudinal orientation.

\subsection{0 | Immunohistochemistry and immunofluorescence}

Colon segments were analyzed with DAB immunohistochemistry of PGP9.5 and Tuj1, and immunofluorescence of GFP and Tuj1. ENCCs in the in vitro experiment were stained for immunofluorescence of p75 neurotrophin receptor (p75 NTR), nestin, and neuronal nuclei (NeuN).

Paraffin-embedded sections were subjected to dewaxing, rehydration, and antigen retrieval using citrate (Beyotime Biotechnology, P0081). The immunohistochemistry procedure was performed according to the manufacturer's instruction of SP Rabbit \& Mouse HRP Kit (Cwbio, CW2069). Immunofluorescence staining started 
from permeabilization using $0.1 \%$ Triton $\mathrm{X}-100$ in PBS for 15 minutes and followed by three times washing in PBS. After this, goat serum (Beyotime, C0265) was used to block for 1 hour. Antibodies (listed in Table. 1) and DAPI reagent were incubated accordingly. All the slides were mounted with antifade mounting medium.

\subsection{1 | Cell lysis and Western blot}

Differentiated ENCCs (see 2.4) were lysed on ice in RIPA buffer (Beyotime Biotechnology, P0013C) containing 1\% protease inhibitor (Roche, 04693116001). Protein quantification was performed using the BCA Protein Assay Kit (Beyotime Biotechnology, P0012). Then, $20 \mu \mathrm{g}$ cell lysate of each sample was boiled after adding laemmli buffer, loaded onto an SDS-PAGE gel, and transferred to a PVDF membrane. Evaporated milk (5\%) in TBS was used as a blocking buffer. After incubation with primary and secondary antibodies, membranes were developed with Immobilon Western chemiluminescent HRP substrate kit (Millipore, WBKLS0500). The antibodies used are listed in Table 1.

(A)
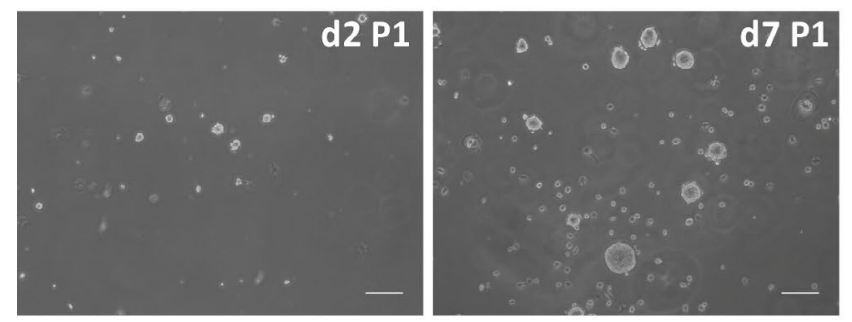

(C)
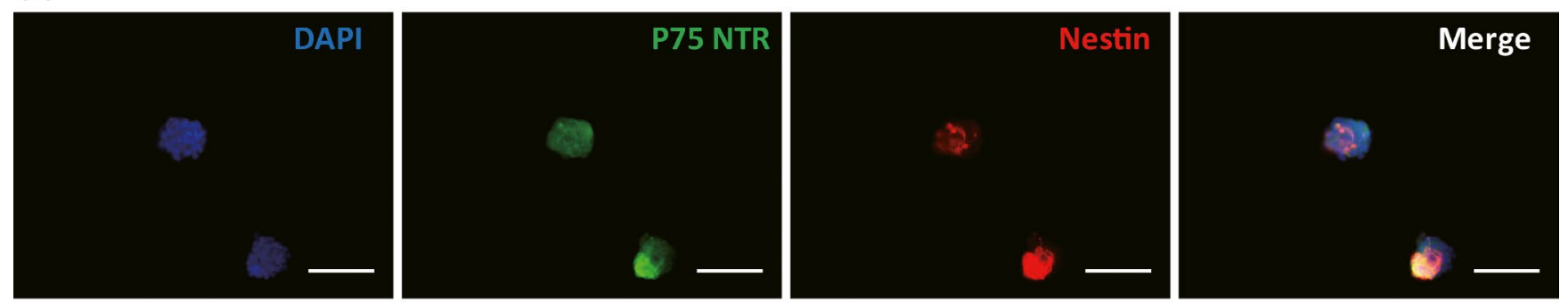

(D)
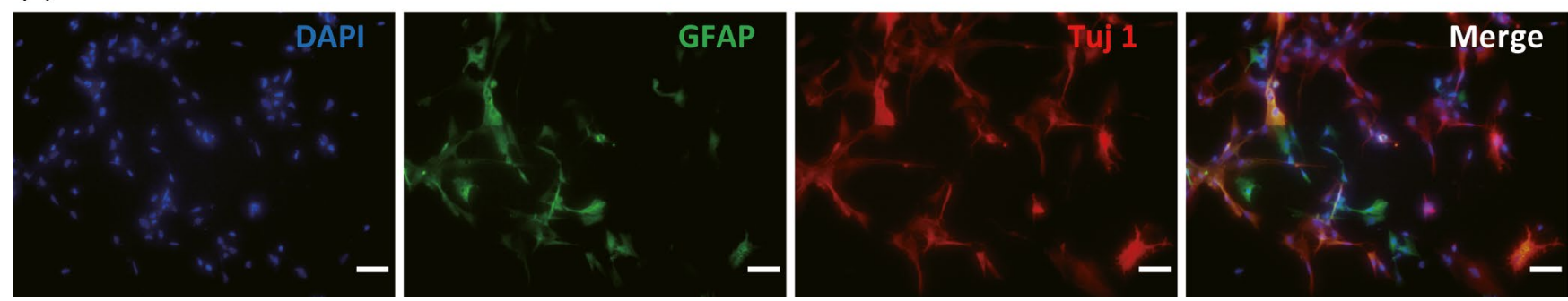

FIGURE 1 Primary culture of ENCC neurosphere and immunofluorescence staining (A) Irregular free-floating ENCC neurospheres appeared on day two. Larger and compacted neurospheres formed on day seven. B, $7 \mathrm{~d}$ after splitting, cell clusters obtained by gentle pipetting (left) showed better neurosphere formation than single-cell suspension obtained via trypsinization with repeated pipetting (right). The latter were more likely to attach and demonstrate morphologic changes. C, Immunofluorescence staining showed considerable expression of neural stem cell markers P75 NTR and Nestin in neurospheres. D, By differentiation induction, Tuj1 positive or GFAP positive cells were derived, indicating neuron and glia. Scale bar, $100 \mu \mathrm{m}$ 


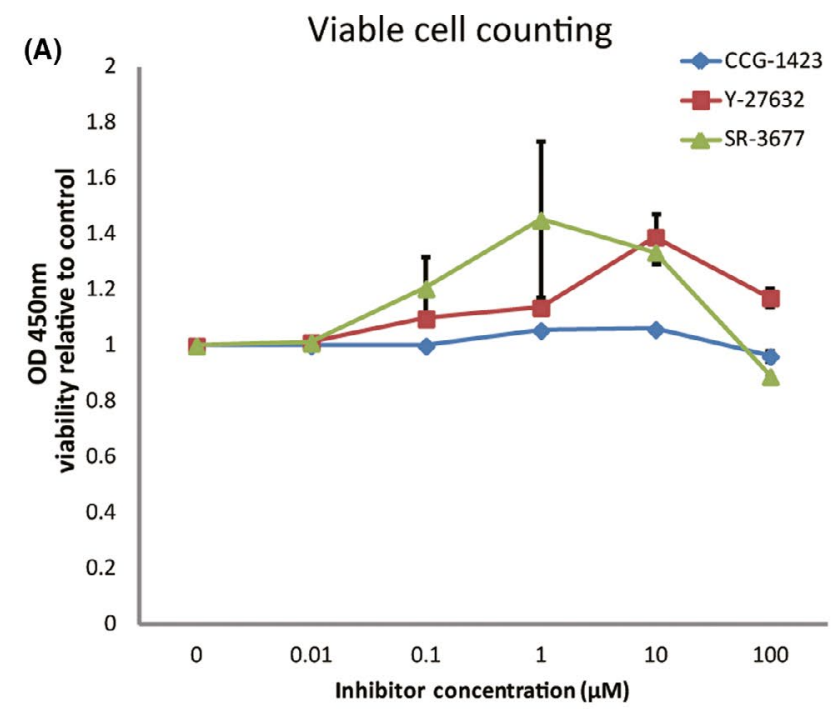

(B) Peak value of viable cell counting

(C)

control

CCG-1423

Y-27632

SR-3677

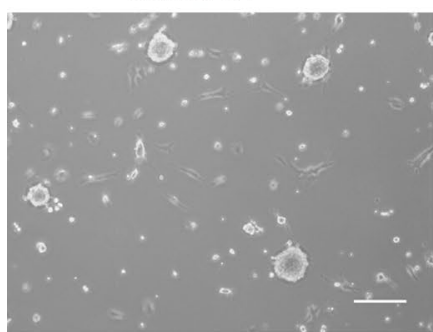

(D)

잉

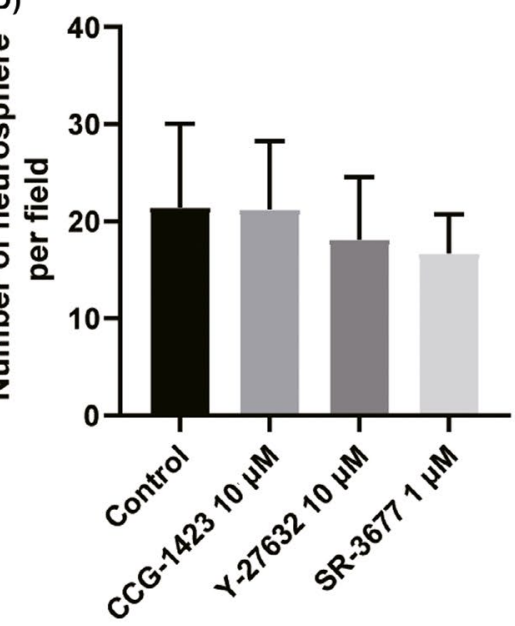

(E)

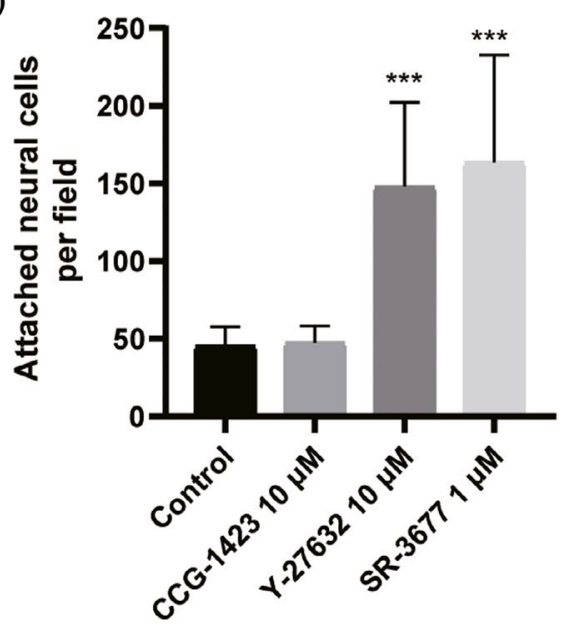

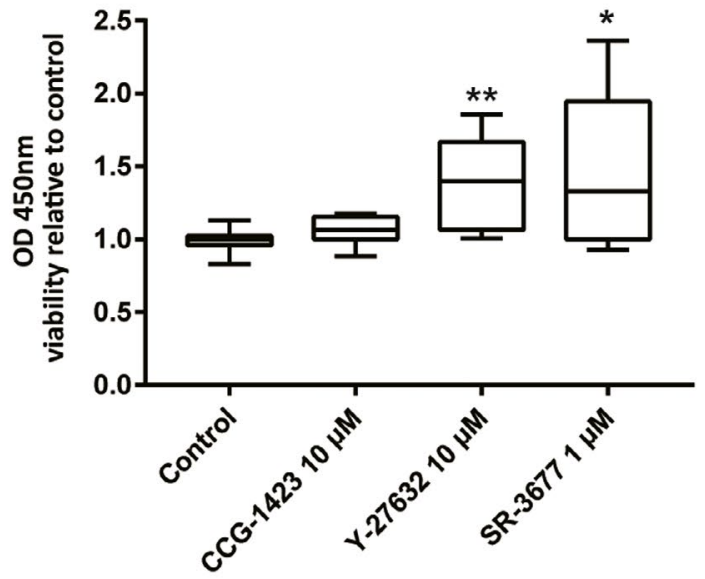
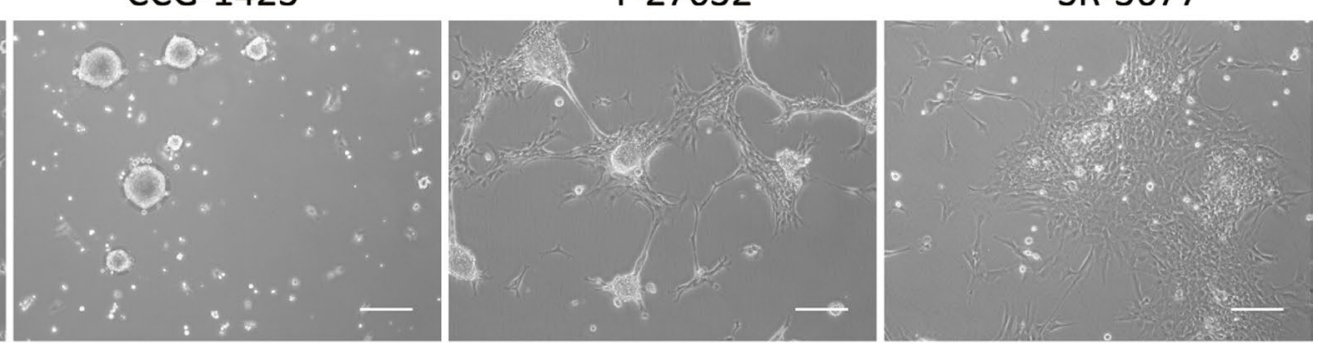

(G)
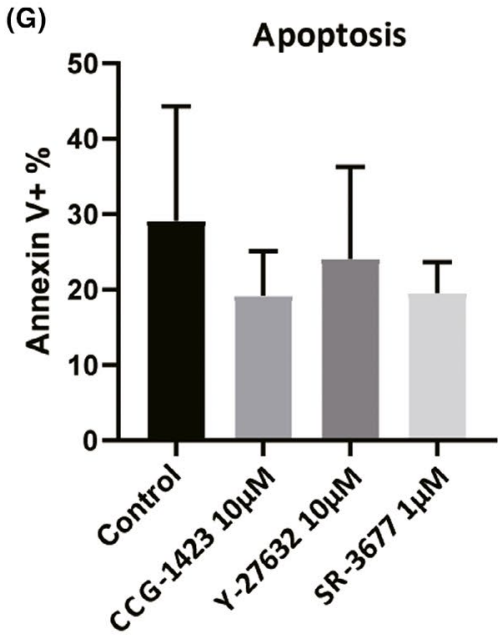

(F) control

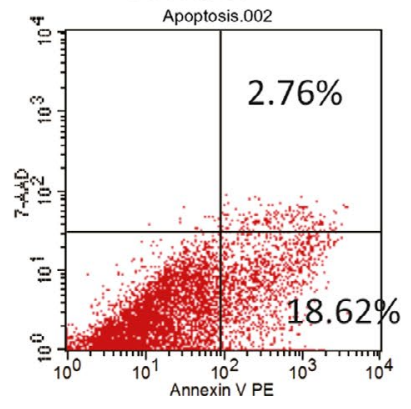

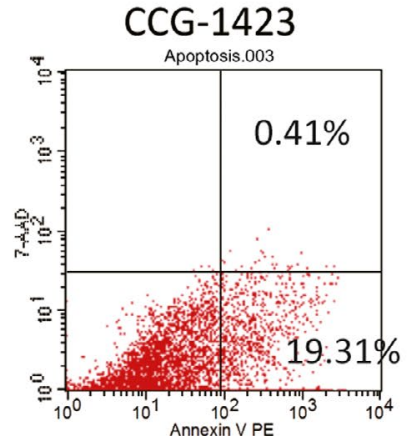

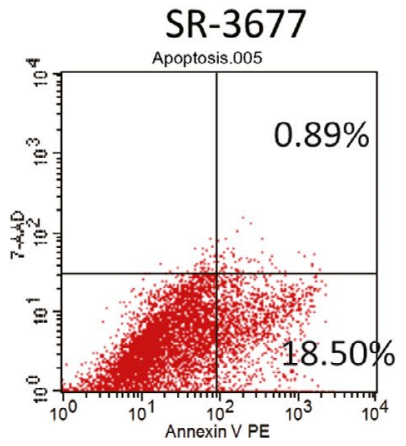


FIGURE 2 Viability, morphology, and apoptosis of ENCCs responded to RhoA/ROCK inhibitors (A) Results of Cell Counting Kit-8 test normalized by non-treated control cells showed different effects on cell viability and growth by each inhibitor at different concentrations. B, treatment with $10 \mu \mathrm{mol} / \mathrm{L}$ Y-27632 and $1 \mu \mathrm{mol} / \mathrm{L}$ SR-3677 showed a significant increased viability of ENCC compared to PBS treated control, and no significant difference was detected upon treatment with $10 \mu \mathrm{mol} / \mathrm{L} \mathrm{CCG}-1432$ ( $t$ test, $\mathrm{X} \pm \mathrm{SD}$, Box-\&Whiskers chart, $\mathrm{n}=24$, $10,10,10)$. C, Representative images of ENCCs grown in different inhibitor groups after $5 \mathrm{~d}$ of treatment. The CCG-1432 group showed similar morphology to that of the control, the other two groups showed some morphological changes of attachment. Scale bar, $100 \mu \mathrm{m}$. $D$, Quantification of the number of neurospheres (shown in panel C) showed no differences between groups ( $t$ test, $X \pm S D, n=11,10,10$, 10). E, Quantification of the number of attached neural cells (shown in panel C) shows that significant more cells were attached in groups treated with $\mathrm{Y}-27632$ or SR-3677 ( $t$ test, $\mathrm{X} \pm \mathrm{SD}, \mathrm{n}=11,10,10,10$ ). F, G, Flow cytometry measurements of apoptosis showed no significant difference between groups ( $t$ test, $X \pm S D, n=3$ )

TABLE 2 Statistical results List of statistical results of ENCCs in vitro experiment

\begin{tabular}{|c|c|c|c|c|c|c|}
\hline Fig. & Assay & $t$-test & Control & CCG-1423 $10 \mu \mathrm{mol} / \mathrm{L}$ & $\mathrm{Y}-2763210 \mu \mathrm{mol} / \mathrm{L}$ & SR-3677 $1 \mu \mathrm{mol} / \mathrm{L}$ \\
\hline Figure 2B & CCK-8 kit & $P$-value & - & .0517 & $.0021^{* *}$ & $.0247^{*}$ \\
\hline \multirow[t]{2}{*}{ Figure 2D } & \multirow[t]{2}{*}{ Neurosphere number } & $X \pm S D$ & $21.45 \pm 8.595$ & $21.20 \pm 7.052$ & $18.10 \pm 6.437$ & $16.70 \pm 4.001$ \\
\hline & & $P$-value & - & .9420 & .3281 & .1269 \\
\hline Figure $2 \mathrm{E}$ & Attached neural cells & $X \pm S D$ & $45.45 \pm 12.44$ & $47.30 \pm 10.87$ & $148.1 \pm 54.16$ & $163.7 \pm 68.91$ \\
\hline \multirow[t]{2}{*}{ Figure $2 \mathrm{G}$} & \multirow[t]{2}{*}{ Apoptosis assay } & $X \pm S D$ & $29.14 \pm 15.12$ & $19.20 \pm 5.902$ & $24.05 \pm 12.16$ & $19.57 \pm 4.028$ \\
\hline & & $P$-value & - & .3484 & .6732 & .3492 \\
\hline \multirow[t]{2}{*}{ Figure $3 C$} & \multirow{2}{*}{$\begin{array}{l}\text { Differentiation } \\
\text { Cell number }\end{array}$} & $X \pm S D$ & $13.84 \pm 1.514$ & $13.24 \pm 4.336$ & $86.71 \pm 19.38$ & $90.66 \pm 23.56$ \\
\hline & & $P$-value & - & .2605 & $<.0001^{* * * *}$ & $<.0001^{* * * *}$ \\
\hline Figure $3 \mathrm{~F}$ & WB Tuj1/ $\beta$-actin & $P$-value & - & .2948 & $.0028^{* *}$ & $.0199^{*}$ \\
\hline \multirow[t]{2}{*}{ Figure 3G } & \multirow[t]{2}{*}{ WB GFAP/ $\beta$-actin } & $X \pm S D$ & $1.000 \pm 0.000$ & $0.9078 \pm 0.2225$ & $1.219 \pm 0.2150$ & $1.306 \pm 0.2034$ \\
\hline & & $P$-value & - & .3565 & $.0316^{*}$ & $.0042^{* *}$ \\
\hline \multirow[t]{2}{*}{ Figure 31} & \multirow[t]{2}{*}{ Migration assay } & $X \pm S D$ & $1.000 \pm 0.3645$ & $0.9147 \pm 0.3062$ & $2.905 \pm 1.647$ & $1.403 \pm 1.373$ \\
\hline & & $P$-value & - & .4040 & $.00004^{* * * *}$ & .2158 \\
\hline
\end{tabular}

CCK-8, Cell Counting Kit-8.

NeuN, neuron nuclear protein.

Tuj1, $\beta$-III-tubulin.

GFAP, glial fibrillary acidic protein.

${ }^{*} P<.05$.

${ }^{* *} P<.01$.

${ }^{* * *} P<.001$.

****P $<.0001$

from gentle pipetting (left) showed better neurospheres' formation than single-cell suspension obtained from trypsinization with repeated pipetting (right). This stressed the importance of minimizing the duration of incubation with dissociative reagents and gentle pipetting.

Immunofluorescence for neural stem cell markers P75 neurotrophin receptor (P75NTR) and nestin showed double-positive staining of the neurospheres (Figure $1 \mathrm{C}$ ). By inducing differentiation, cells became beta-III-tubulin (Tuj1) positive or glial fibrillary acidic protein (GFAP) positive, indicating neuronal or glial differentiation (Figure 1D). Cells co-expressed with Tuj1 and GFAP were also showed but the mechanism remained unclear. Premature stage of the cells or multi-differentiation potential could be plausible explanation, while further studies required. ${ }^{23,24}$ Taken together, these results showed that we were able to efficiently generate ENCC neurospheres, which could be used for following analyses.

\section{2 | RhoA/ROCK inhibitors increased ENCCs viability}

To assess the effect of inhibiting the RhoA/ROCK pathway on ENCC viability, cells were treated with different concentrations of RhoA inhibitor CCG-1423, ${ }^{25}$ ROCK inhibitor Y-27632 dihydrochloride, ${ }^{26}$ and ROCK2 inhibitor SR-3677, ${ }^{27}$ and then subjected to a viable cell counting assay. Since this assay was directly proportional to the number of living cells, it could reflect how cell grew 
and proliferated. Results showed that treatment with CCG-1423 did not affect the number of live cells, while treatment with Y-27632 and SR-3677 showed a remarkable increase when compared to control (Figure 2A, B; Table 2). Both Y-27632 and SR-3677 enhanced viable cell numbers at a concentration of $0.1 \mu \mathrm{mol} / \mathrm{L}$, with a peak at $10 \mu \mathrm{mol} / \mathrm{L}$ for $\mathrm{Y}-27632$ and $1 \mu \mathrm{mol} / \mathrm{L}$ for SR-3677, respectively. Upon taking the peak value of each group, significant increase caused by Y-27632 and SR-3677 was confirmed (Figure 2B; Table 2).
(A)

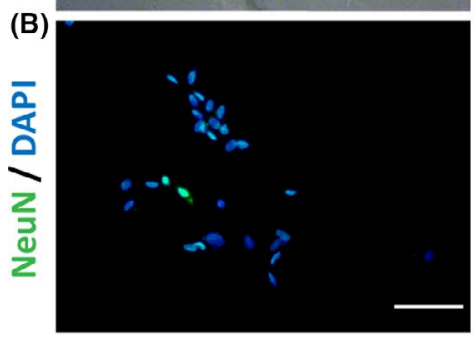

(C)

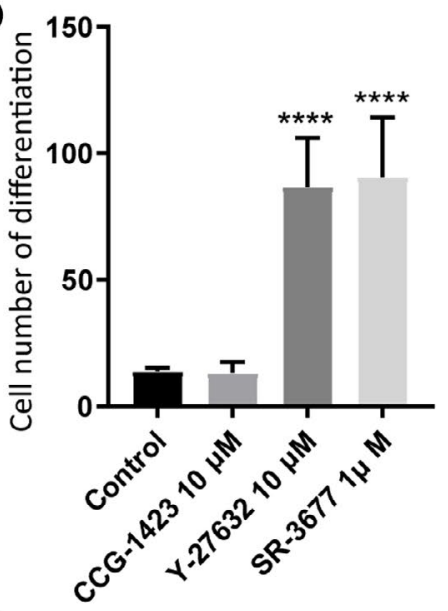

(F)

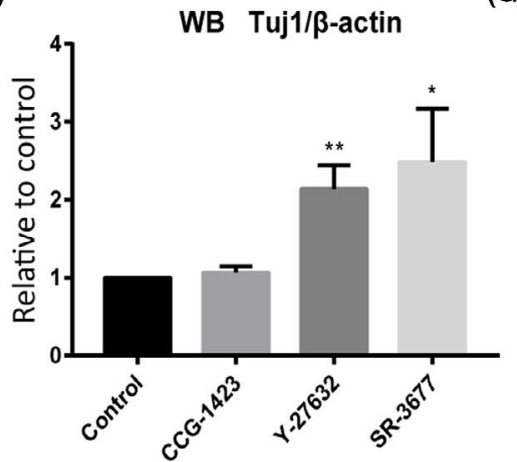

(H)

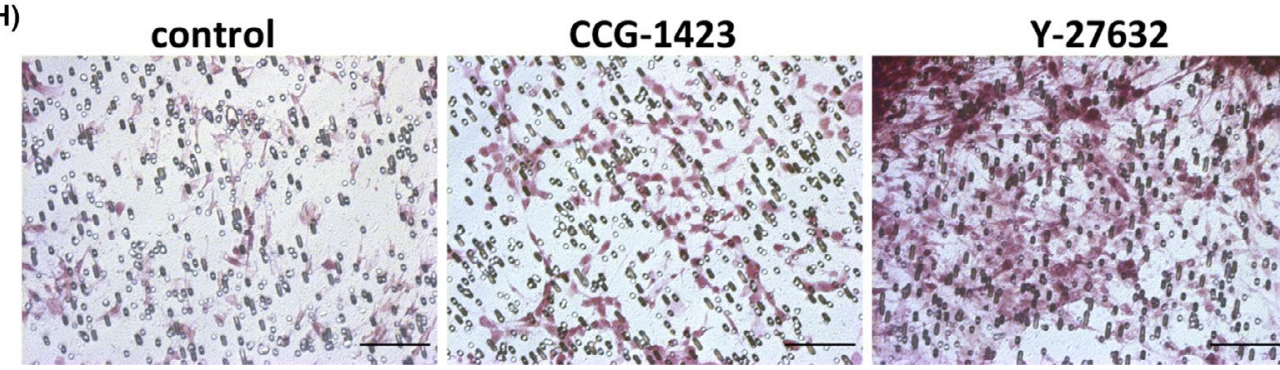

CCG-1423
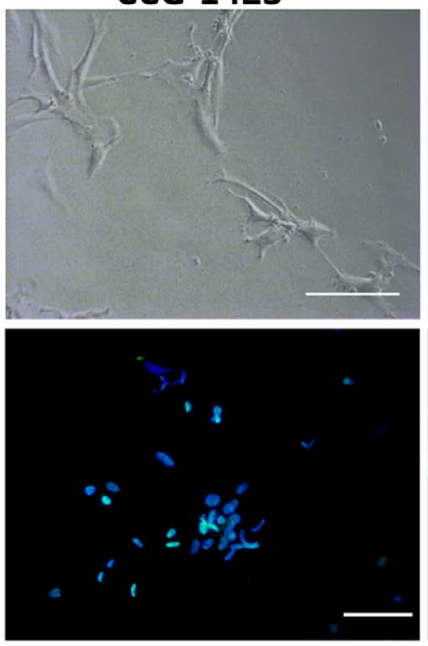

(D)

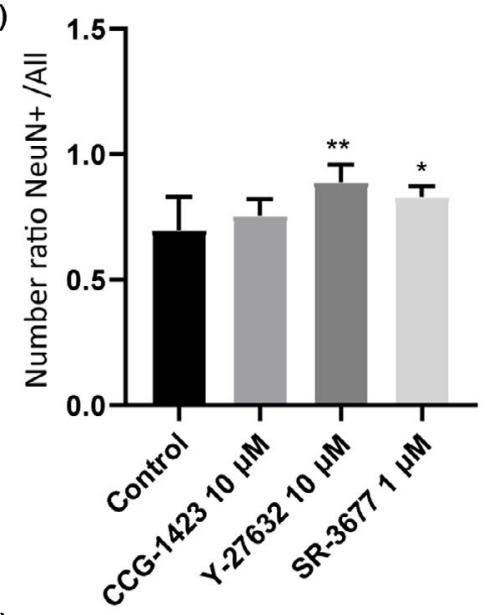

(G)

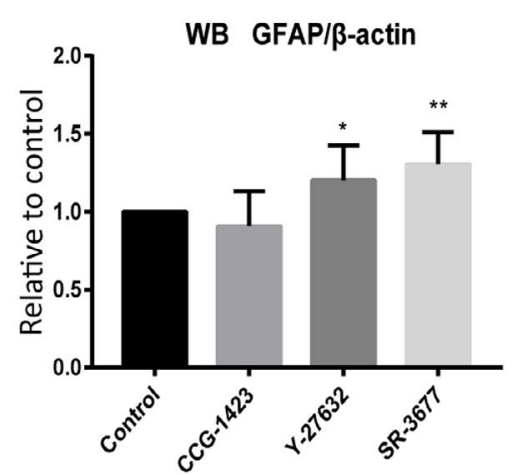

Y-27632
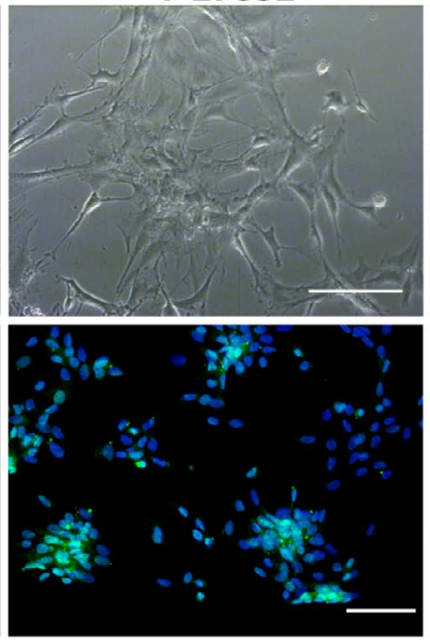

(E)

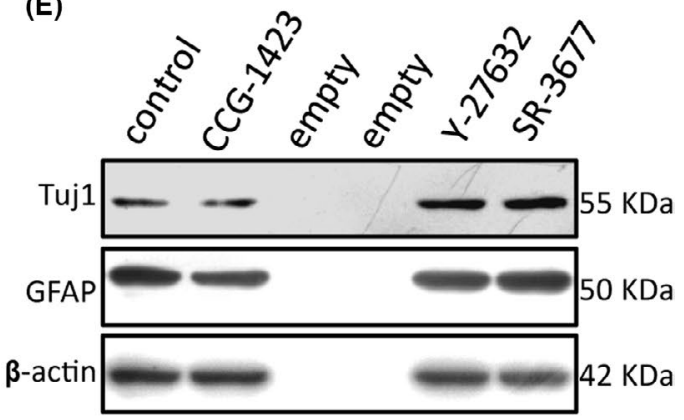

SR-3677

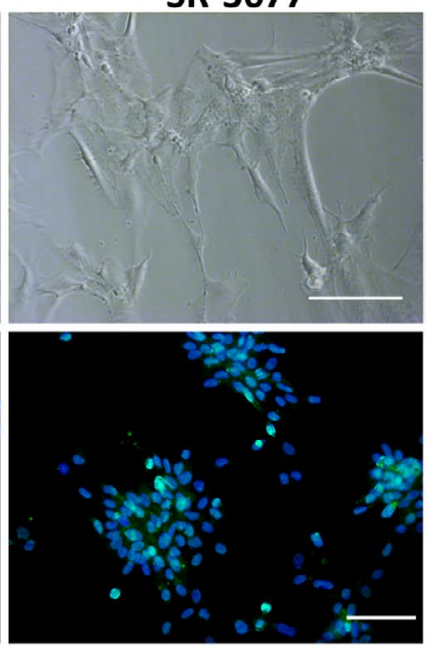

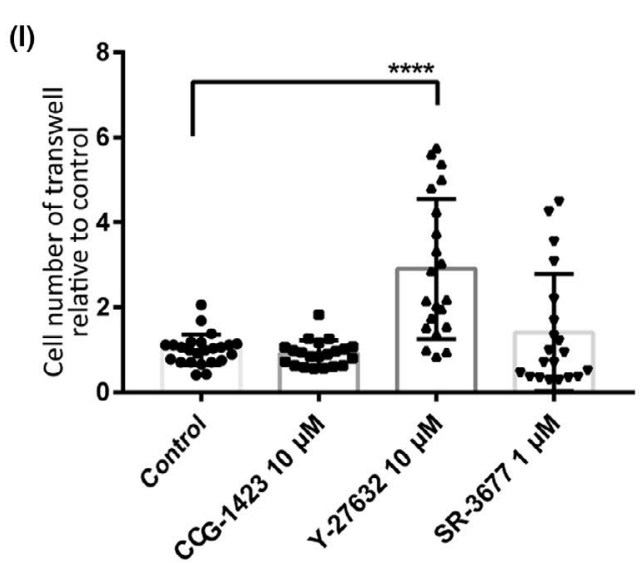

CCG-1423

Y-27632

SR-3677

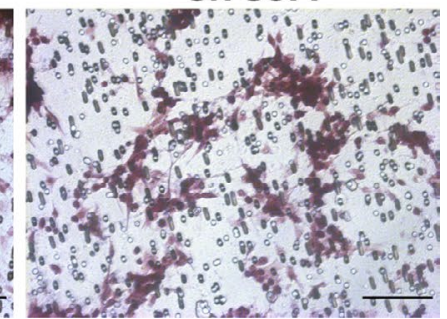


FIGURE 3 Differentiation and migration of ENCCs responded to RhoA/ROCK inhibitors (A, B) The differentiation behavior of ENCCs under light microscopy and immunofluorescence staining of neuronal nuclei (NeuN). C, The number of differentiated ENCCs quantified per light microscope field (shown in panel A). ENCCs treated with Y-27632 or SR-3677 showed a significantly more differentiated cells compared to the control group and the group treated with CCG-1432 ( $t$ test, $X \pm S D, n=12$ ). D, The percentage of NeuN ${ }^{+}$cells quantified per field of view (shown in panel B) was higher in groups treated with $Y-27632$ and SR-3677 than in the other two groups $(t$ test, $X \pm S D, n=9)$. $E$, Western blot analysis of differentiated ENCCs showed increased of Tuj1 and GFAP protein expressed in ENCCs treated with Y-27632 and SR-3677 compared to control and CCG-1423 group. F, Quantification of the Western blot quantification of Tuj1 relative to $\beta$-actin ( $t$ test, $X \pm S D, n=3$ ). G, Quantification of the Western blot of GFAP relative to $\beta$-actin ( $t$ test, $X \pm S D, n=6$ ). $H$, Trans-well migration assay assessed by the number of ENCCs that migrated through the chamber membrane and stained with gentian violet. The distribution of migrated ENCCs showed a homogenous pattern in the control, CCG-1432, and Y-27632 groups, while a non-uniform pattern was observed in the SR-3677 group. I, Quantification of migrated cells per imaged field normalized by control showed a significant increase in $\mathrm{Y}-27632$ treated group $(t$ test, $X \pm S D, n=24,21,21,20)$. Scale bar, $100 \mu m$

Based on these findings, the optimal doses, $10 \mu \mathrm{mol} / \mathrm{L}$ for $\mathrm{Y}-27632$ and $1 \mu \mathrm{mol} / \mathrm{L}$ for SR-3677, were chosen for further experiments. Although no significant difference was observed upon treatment with CCG-1423, we decided to include this inhibitor at a concentration of $10 \mu \mathrm{mol} / \mathrm{L}$ in further experiments.

For morphology features, ENCCs neurospheres showed various morphological behaviors upon treatment with different inhibitors (Figure 2C). As quantified by the number of neurospheres and attached cells, CCG1423 showed similar results as that observed in control. However, the Y-27632 and SR-3677 groups revealed comparable neurosphere amounts but significant higher induction of cell attachment (Figure 2D, E; Table 2).

In order to detect whether the different inhibitors influenced apoptosis, FACS analysis with Annexin V: PE Apoptosis Detection Kit was used (Figure 2F). No significant difference was detected between the three treated groups and the control (Figure 2G). All statistical results have been shown in Table 2 .

\subsection{RhoA/ROCK inhibitors induced the differentiation and migration of ENCCs}

ENCC possesses vital features to differentiate into both neurons and glia, which is essential for the formation of a proper ENS network. Therefore, it is important to investigate whether the inhibitors influence the differentiation ability of single cells obtained from ENCCs. To study this, cells were treated with $10 \mu \mathrm{mol} / \mathrm{L}$ CCG-1423, $10 \mu \mathrm{mol} / \mathrm{L}$ Y-27632, and $1 \mu \mathrm{mol} / \mathrm{L}$ SR-3677 for 72 hours, accompanied by $2 \%$ FCS stimulation. Our results showed that in all groups, ENCC differentiation occurred, which could be observed by acquiring a longitudinal shape and emergence of neurites (Figure 3A). Immunofluorescence staining showed the expression of the neuronal marker neuronal nuclei $(\mathrm{NeuN})$ in differentiated cells (Figure 3B). Quantification of differentiated cells numbers (Figure 3A, C; Table 2) and percentages of $\mathrm{NeuN}^{+}$ cells (Figure 3B, D; Table 2) showed a significant increase in the number of cells that were differentiated upon treatment with either Y-27632 or SR-3677, compared to cells treated with CCG-1423 and the PBS treated controls. In addition, Western blot analysis confirmed these findings showing increased expression of Tuj 1 and GFAP upon treatment with either Y-27632 or SR-3677 (Figure 3E-G; Table 2). According to the results, Y-27632 and SR-3677 could not only accelerate the total number of differentiated cells by promoting differentiation ability, but also increase the proportion of differentiated neurons and glial cells.
To analyze the effect of the RhoA/ROCK inhibitors on cell migration, a trans-well assay was performed where the three different inhibitors were added to both the upper and bottom chambers. Our results showed that treatment with Y-27632 significantly increased the migration rate of ENCCs, while the other two inhibitors had no effect (Figure $3 \mathrm{H}, \mathrm{I})$. Meanwhile, we noticed that cells treated with SR-3677 displayed a heterogeneous migration pattern, in which regions of cell aggregated and empty regions were randomly distributed in the membrane, exhibiting a large standard deviation (Figure 3H, l; Table 2). The statistical results of all the above assays are shown in Table 2.

\subsection{ENCC transduction required a proper lentiviral multiplicity of infection (MOI) to balance transduction efficiency and neurosphere morphology}

For cell-based therapy, labeling the cells to be grafted could ensure continuous tracking after transplantation in the animal model. Lentiviral transduction enables efficient, stable, and long-term labeling of ENS stem cells that is retained after transplantation into the gut in vivo ${ }^{28}$ and does not induce toxicity. ${ }^{29}$ Moreover, the lentiviral approach could offer the possibility to transfect with a construct containing specific genes of interest in future experiments, such as genes containing mutations or rescue constructs for gene therapy. ${ }^{28}$

For our experiments, we decided to use a GFP-expressing lentiviral construct and started by determining the optimal multiplicity of infection (MOI) among 5×, 25×, 125×, and 250×. Cells were inspected microscopically to assess their morphological features and analyzed by FACS to determine the transduction efficiency. Transduction with the lowest amount of virus, $5 \times \mathrm{MOI}$, provided insufficient labeling, whereas transduction with $125 \times \mathrm{MOI}$ and $250 \times \mathrm{MOI}$ influenced the shape of neurospheres as they appeared elongated (Figure 4A). 25× MOI seemed to be the optimal lentiviral concentration that resulted in adequate transduction efficiency (85\% GFP-positive ENCCs), while maintaining the original shape of ENCCs (Figure 4B, C). After transduction with $25 \times \mathrm{MOI}$, neurospheres were collected, cultured, and further differentiated. We were able to see that the GFP gene could be successfully transferred to the daughter cells and retained through differentiation process (Figure 4D), indicating that good labeling of ENCCs could be obtained with this approach. 


\section{5 | Y-27632 improved the efficiency of ENCC transplantation in a hypoganglionic rat model}

Considering that the ROCK inhibitor $Y$-27632 gave the most comprehensive results in our in vitro assays from the three RhoA/ROCK inhibitors tested, we expected that Y-27632 would have the potential to increase the efficiency of ENCC transplantation in vivo. To test this hypothesis, we performed ENCC transplantation in an inducible hypoganglionic rat model established by 30 minutes incubation with $0.1 \%$ benzalkonium chloride (Figure $5 \mathrm{~A}$ ). It is a widely used non-genetic modulated HSCR model, and ideal hypoganglionic segments could be achieved within a limited time. ${ }^{30,31}$

To ensure that a hypoganglionic gut was induced, histologic evaluation of the BAC-treated segment was performed 2, 4, and 6 weeks later. At these three time points, stenosis of the treated segment, and proximal dilation was macroscopically observed in the model (Figure 5B). Immunostaining of Tuj1 and PGP9.5 was performed as well, and it showed reduction of neural cells after treatment (Figure 5C). Quantified results showed a significant decrease in the number (Figure 5D, $X \pm$ SEM, control $5.000 \pm 0.4629,2$ weeks $2.636 \pm 0.2787$, 4 weeks $3.400 \pm 0.2667,6$ weeks $3.600 \pm 0.4522$; $P$-value: $P$ - 2 weeks $=.0002^{* * *}$,
P-4 weeks $=.0063^{* *}, P-6$ weeks $=.0480^{*}$ ) and size (Figure 5E , $X \pm$ SEM, control $1.000 \pm 0.1520,2$ weeks $0.5189 \pm 0.0862$, 4 weeks $0.5311 \pm 0.0763$, 6 weeks $0.5025 \pm 0.0703$; $P$-value: $P$ - 2 weeks $=.0078^{* *}$, $P$-4 weeks $=.0078^{* *}, P-6$ weeks $=.0044^{* *}$ ) of the ganglia after BAC treatment. Thus, hypoganglionic models were successfully induced and could be used for ENCC transplantations.

Transplantation experiments had a total duration of 6 weeks (Figure 6A), which consisted of the establishment of the hypoganglionic rat model ( 3 weeks), and in parallel the culturing and GFPlabeling of ENCCs. Then, at day 21, ENCCs were transplanted into the muscle layer by injections at the clock positions 3, 6, 9, and 12 (Figure 6B), followed by postoperative treatment with either PBS or Y-27632 every alternate day until day 42. During the experiment, the bodyweight of all the rats was measured every other day and analyzed as the relative ratio of weight change to initial weight (Figure 6C). ANOVA test revealed that the bodyweight changed over time $(P<.0001)$ showing two obvious decreases after two surgeries. However, between the three groups, no significant difference $(P=$.7349) was found.

Three weeks after transplantation, 17 rats from the control group, 18 rats in the ENCC-only group, and 18 rats in the ENCC + Y-27632

(A)
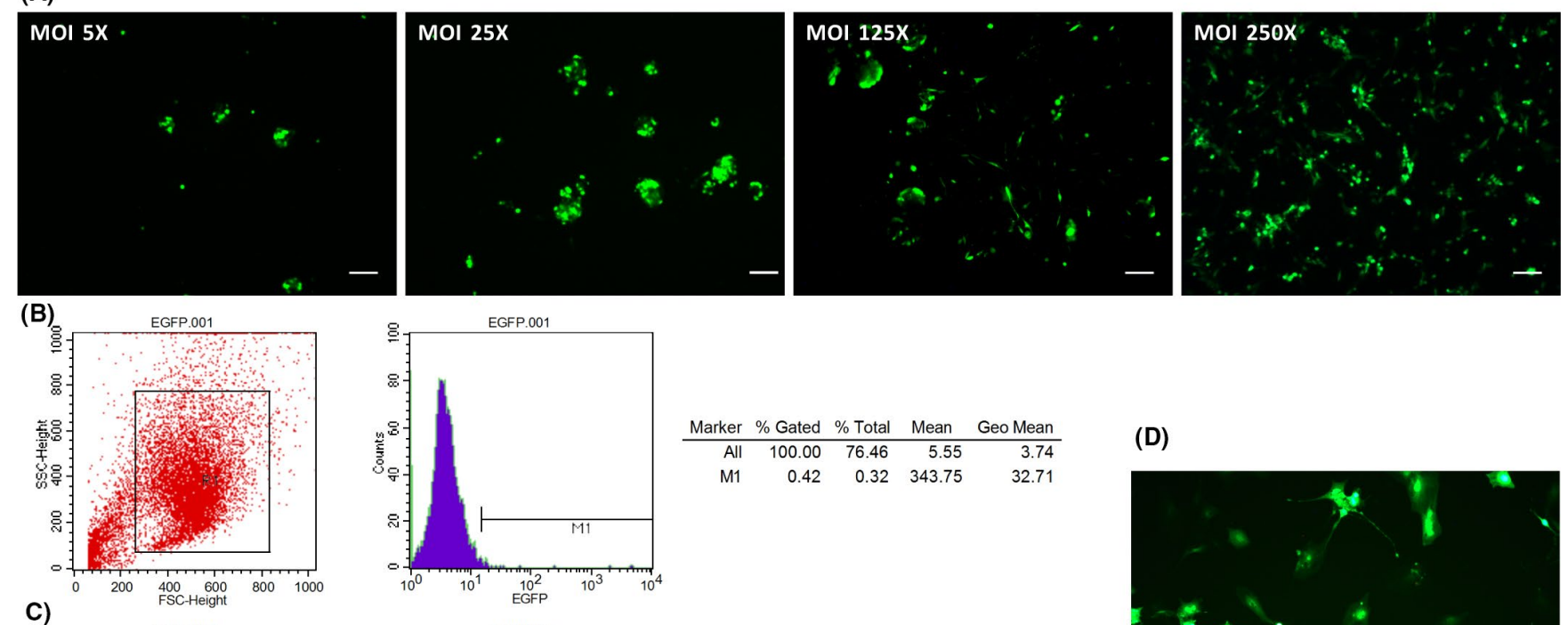

(D)
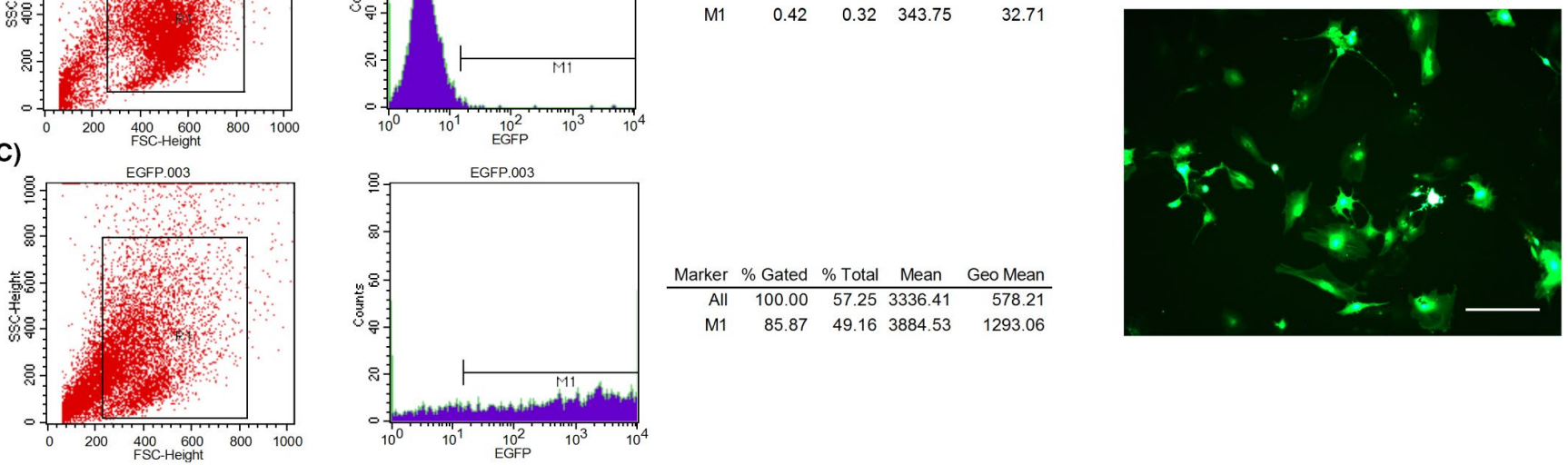

FIGURE 4 ENCCs labeled by lentiviral transduction of GFP (A) Lentiviral transduction of GFP into ENCCs using different multiplicity of infection (MOI) showed that $25 \times \mathrm{MOI}$ showed the proper ratio to balance transduction efficiency and retention of proper morphology. $5 \times$ $\mathrm{MOI}$ was insufficient for labeling, while $125 \times$ and $250 \times \mathrm{MOI}$ would trigger changes to cell shape. B, Negative control for flow cytometry analysis of transduction efficiency. C, Transduction efficiency quantification by flow cytometry showed that upon transduction with $25 \times$ MOI, 85.87\% of the ENCCs were GFP-positive. D, Representative image of transduced ENCCs after second passage demonstrated that GFP was incorporated, passed on to next-generation cells, and retained through the differentiation process. Scale bar, $100 \mu \mathrm{m}$ 
(A)

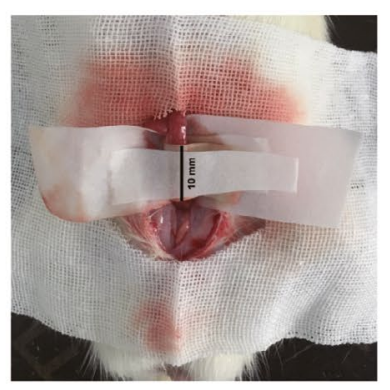

(C)

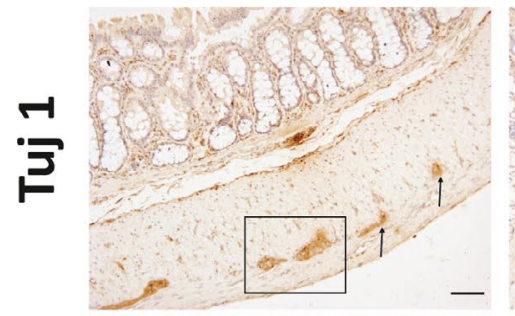

ำ

(D)

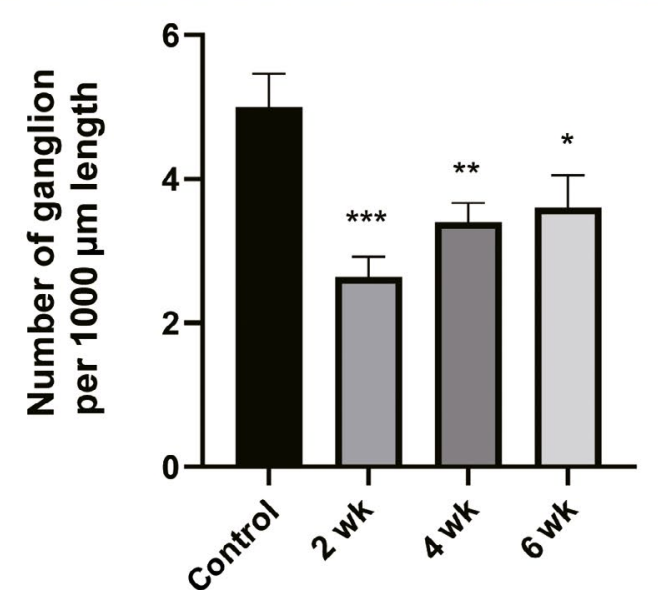

(B)

2 wk
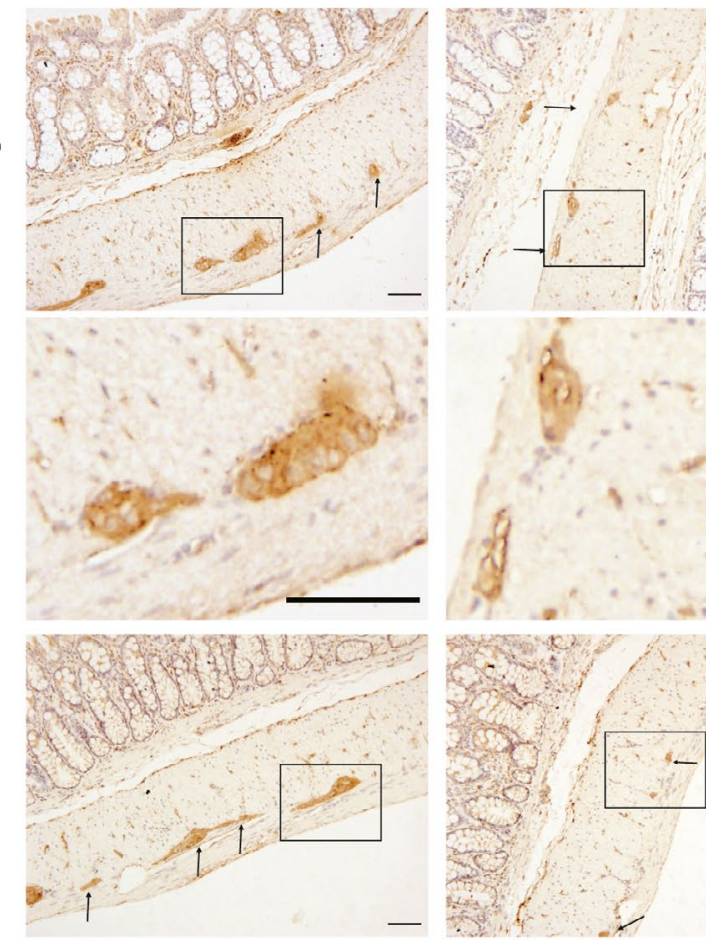
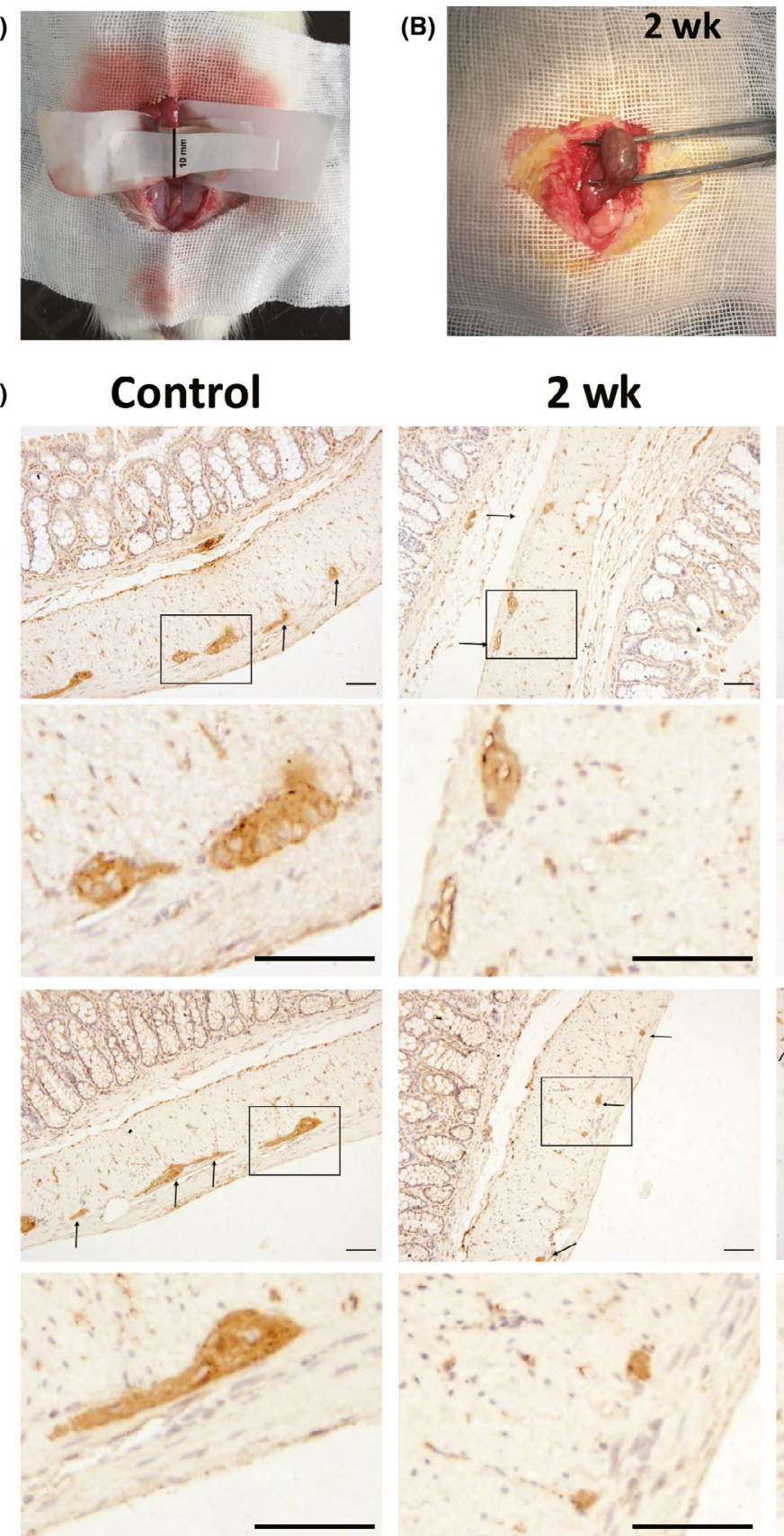

4 wk
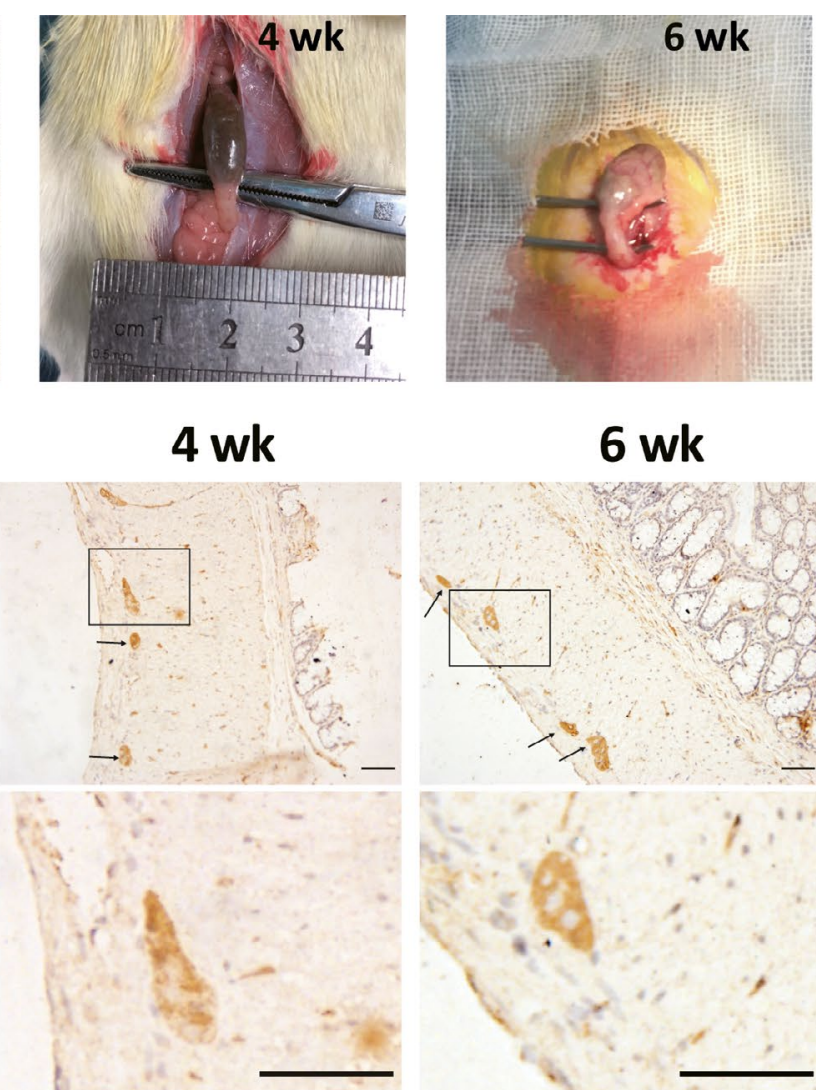

6 wk
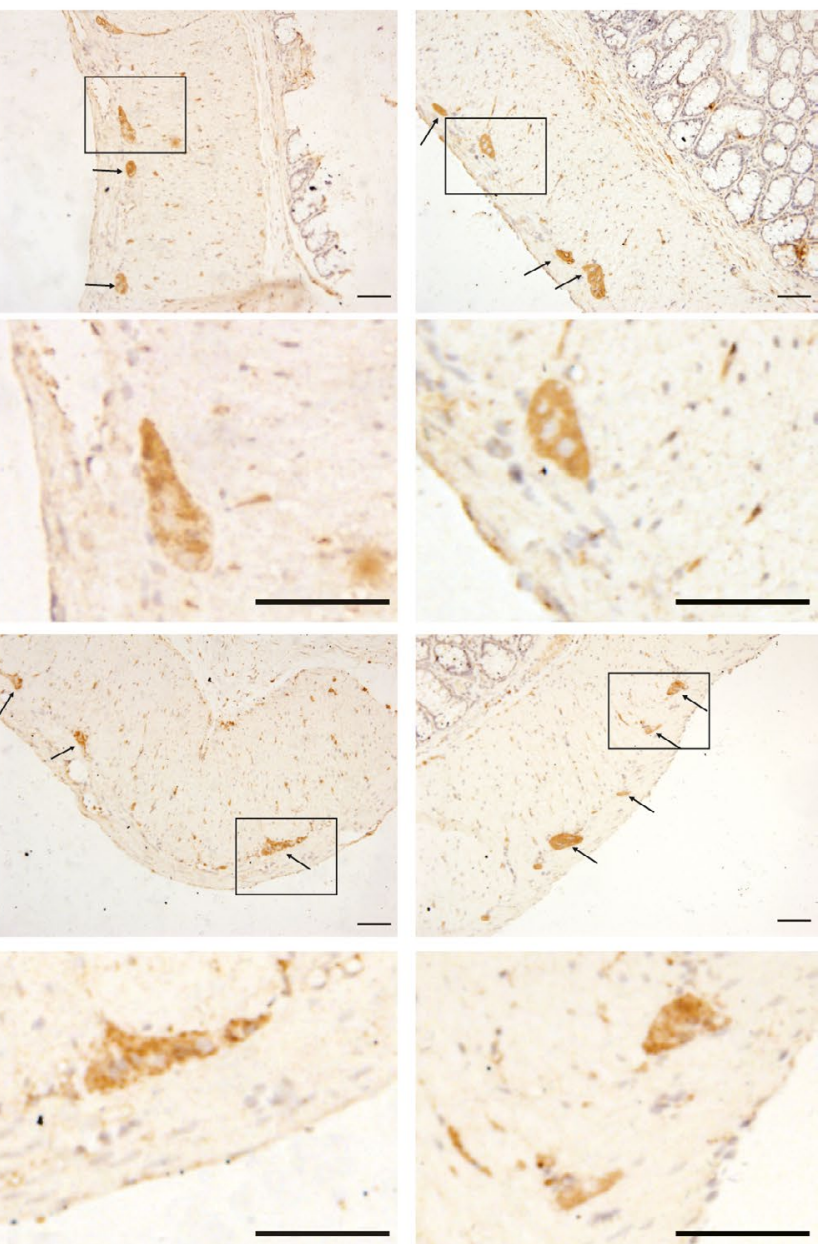

(E)

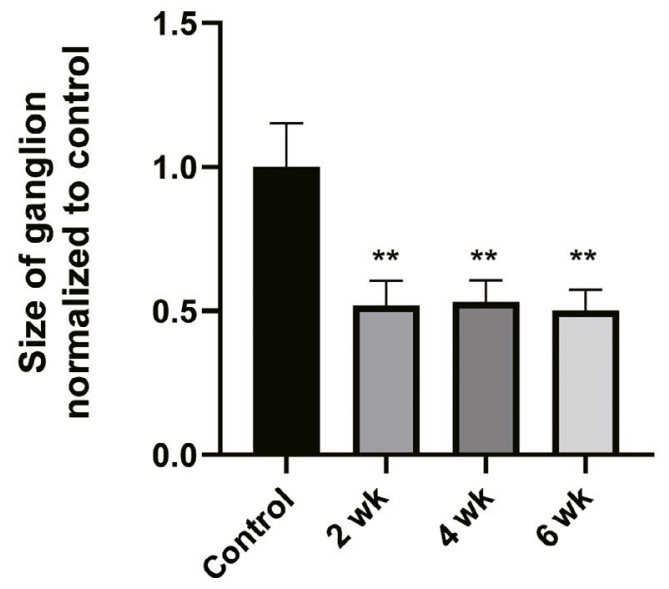


FIGURE 5 Establishment and evaluation of the induced hypoganglionic rat model (A) The hypoganglionic rat model was established by incubating the target colon segment in $0.1 \%$ benzalkonium chloride for $30 \mathrm{~min}$. B, At 2, 4 and 6 wks after surgery, the rats showed stenosis change of the treated segment and dilation of the proximal colon. C, Immunohistochemical staining of the treated segments by Tuj 1 and PGP9.5 showed hypoganglionosis, which was quantified in panel D and E. Scale bar, $100 \mu \mathrm{m}$. D, E, Ganglion's number and size reduced at 2 , 4 , and 6 wks after BAC treatment ( $t$ test, $X \pm S E M, n=8,11,10,10$ )

group survived. Seven rats died due to anesthesia intolerance or wound biting or bleeding. H\&E staining of the transplanted colon segment in ENCC-only and ENCC + Y-27632 groups showed slight cell aggregation at the injection site (Figure 6D).

To unravel the effect of ROCK inhibitor on ENCC transplantation, double-immunofluorescent staining of GFP and Tuj1 was performed. $\mathrm{GFP}^{+}$cells were scored to determine the number of exogenous (grafted) ENCCs, and GFP ${ }^{-} /$Tuj1 $^{+}$cells were scored to determine other neural cells which consisting mostly of the endogenous ENS cells and a fraction of unlabeled graft ENCCs (Figure 6E). Since Y-27632 was injected intraperitoneally, all above-mentioned cells are subjected to this treatment. Grafted ENCCs survived and migrated longitudinally and/or laterally into the mucosa, submucosa and muscle layer in both ENCC-only and ENCC + Y-27632 group. However, a significantly broader distribution of $\mathrm{GFP}^{+}$cells was detected in the ENCC + Y-27632 group, both in the injection site and adjacent region. Fluorescence density was scored for quantitative analysis (Figure 6F, $\mathrm{X} \pm \mathrm{SEM}$ of GFP $^{+}$, ENCC-only $1.538 \pm 0.1576$, ENCC + Y-27632 $2.315 \pm 0.3148$, $\left.P=.0007^{* * *}\right)$.

As to $\mathrm{GFP}^{-} / \mathrm{Tuj}^{+}$cells, quantification of the fluorescence showed comparable results of control and ENCCs-only groups, but a significantly higher density in the ENCCs + Y-27632 group. (Figure 6F, X \pm SEM of $\mathrm{GFP}^{-} /$Tuj1 $^{+}$, control $1.000 \pm 0.0801$, ENCCsonly $0.9094 \pm 0.1187, \mathrm{ENCCs}+\mathrm{Y}-276321.664 \pm 0.2120, P$ control vs ENCCs-only $=.5310, P$ control vs ENCCs + Y-27632 $=.0073^{* *}$, $P$ ENCCs-only vs ENCCs + Y-27632 $\left.=.0041^{* *}\right)$. It suggested that Y-27632 affected not only the graft ENCCs, but also the endogenous ENS with increased distribution.

To verify these findings, we performed immunostainings using the neuronal marker Tuj1 and the pan-neuronal marker PGP9.5 (essential for HSCR diagnosis), and also performed separate immunofluorescence of GFP (Figure S1A, B). The density of each staining was scored to evaluate total neural cells or graft ENCCs, and similar results as significantly higher density of PGP9.5, Tuj1, and GFP were obtained in the ENCC + Y-27632 group compared to others (Figure S1C-D).

Together, these results showed that Y-27632 could enhance the distribution of the exogenous grafted ENCCs and also benefit endogenous ENS restoration thereby providing an auxiliary contribution. These findings implied that ROCK inhibitor Y-27632 has the potential to increase the efficiency of ENCC-based therapy by enhancing cell survival, neural differentiation, and migratory ability.

\section{4 | DISCUSSION}

In this study, we demonstrated the positive influence of ROCK signaling pathway inhibitors on ENCC viability and growth, differentiation, and migration in vitro. We also showed increased ENCC transplantation efficiency by inhibiting the ROCK signaling pathway in an induced hypoganglionic rat model, indicating the accelerating effect on ENCC-based therapy.

Cell-based therapy is considered to be the most promising therapeutic approach for HSCR, especially since several studies have shown that ENCC-based transplantation could successfully restore the ENS. ${ }^{5-7}$ However, this approach has limitations, such as immune reactions, inflammation, poor survival, limited migration, and apoptosis of the transplanted ENCCs. To solve these issues, the cells used for transplantation must be carefully considered. Both embryonic and postnatal ENCC have been applied for therapeutic transplantation research. Although using postnatal and adult ENS cells for transplantation gave promising results, ${ }^{7,32}$ ENS stem/progenitor cells undergo a decline in their proliferative potential, self-renewal and multi-lineage differentiation capacity from fetal to postnatal stages, ${ }^{33}$ and also in ability to form ENS. Therefore, it may be better to aim at replicating the embryonic ENS stage rather than using postnatal ENS stem/progenitor cells for ENS cell therapies considering these features, ${ }^{34}$ yet this requires further evaluation to balance other influencing advantages and disadvantages of both cell types. Currently, induced pluripotent stem cells (iPSCs) are also considered to be a promising source, as they are autologous, easy to generate and expand. And interestingly, ROCK inhibitor has also been widely used during iPSCs culture to promote growth. ${ }^{35}$ Although differentiation protocols for derivation of neural cells from iPSC have been established by various research groups based on the modulation of known inductive signals, ${ }^{36-38}$ neural cells obtained were

FIGURE 6 ENCC transplantation in the hypoganglionic rat model shows increased grafting ability upon treatment with Y-27632. A, Overview of the experimental timeline. The duration was $6 \mathrm{wks}$, which consisted of rat model establishment, ENCCs culture, GFPlabeling, ENCCs transplantation by injections into the muscle layer, and postoperative treatment with PBS or Y-27632. B, Transplantation was performed by $3,6,9$, and 12 clockwise injections into the muscle layer of the segment center, shown as black arrows. C, Bodyweight measured by relative change ratio to initial weight at day 0 showed changes over time and obvious decreases after the two operations. No significant difference was found between the groups (ANOVA test, $n=17,18,18$ ). D, H\&E staining of the transplanted segment in ENCConly group and ENCC + Y-27632 group showed slight cell aggregation at the injection site but otherwise no obvious structural changes. Scale bar, $200 \mu \mathrm{m}$. E, Double-IF-staining of GFP and Tuj1 in transplanted tissue. GFP ${ }^{+}$cells were indicated by the *, and GFP $/$Tuj $^{+}$cells were indicated by the arrows. Scale bar, $100 \mu \mathrm{m}$. F, Fluorescence densities were scored and showed a significant increase in presence of both $\mathrm{GFP}^{+}$and $\mathrm{GFP}^{-} /$Tuj $^{+}$cells in the ENCC + Y-27632 group ( $t$ test, $\mathrm{X} \pm \mathrm{SEM}, \mathrm{n}=17,18,18$ ) 
(A)

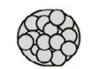

ENCCs culture Lentiviraltransdution

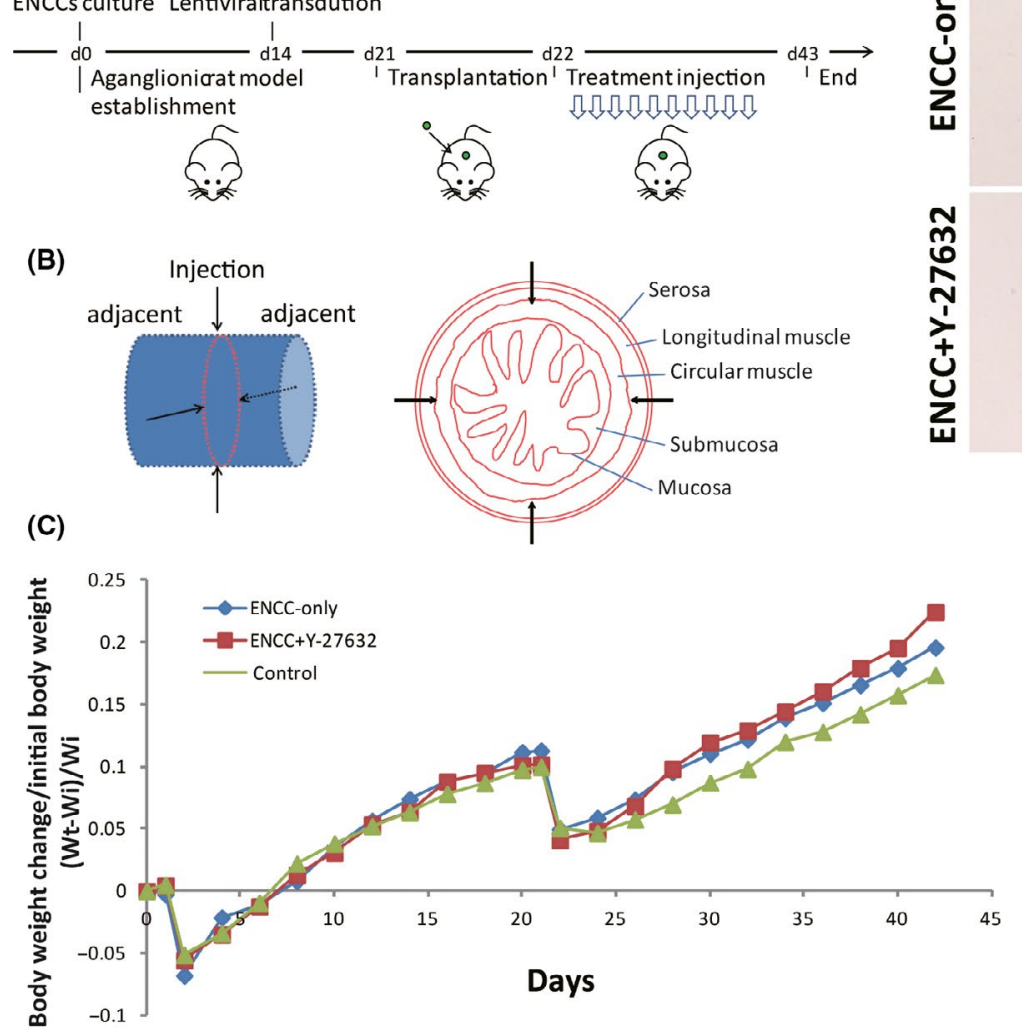

(E) Control

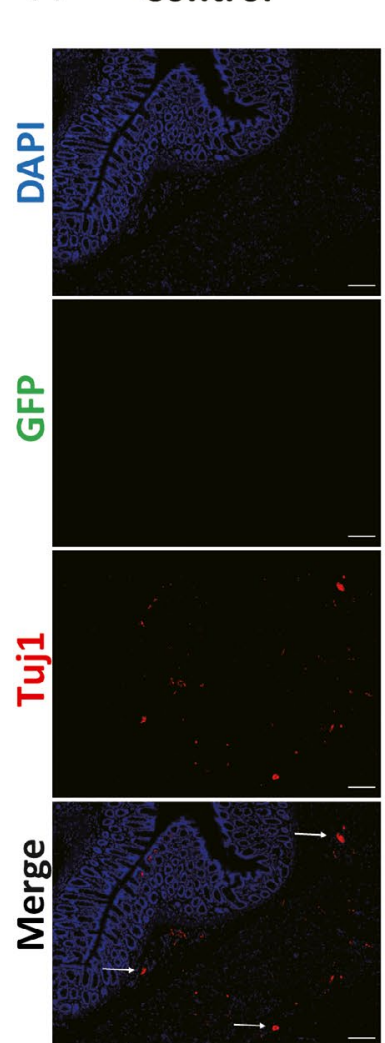

(D)

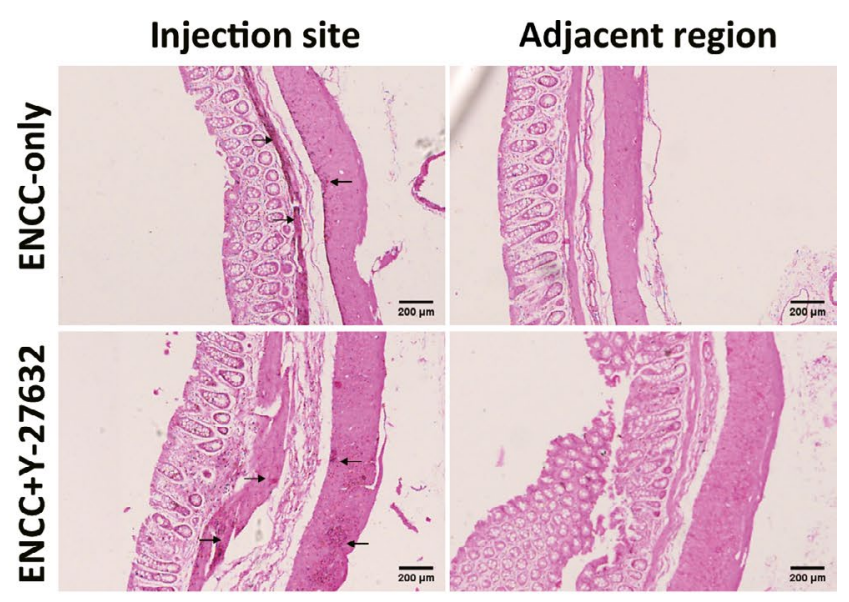

(F)

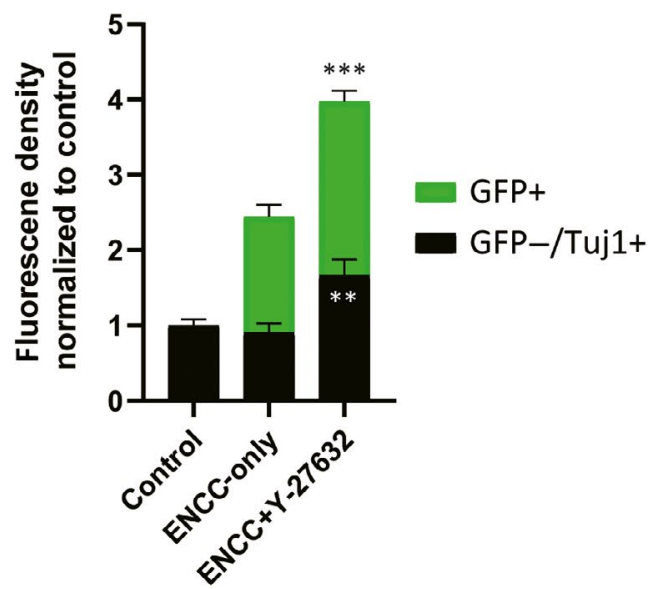

ENCC+Y-27632

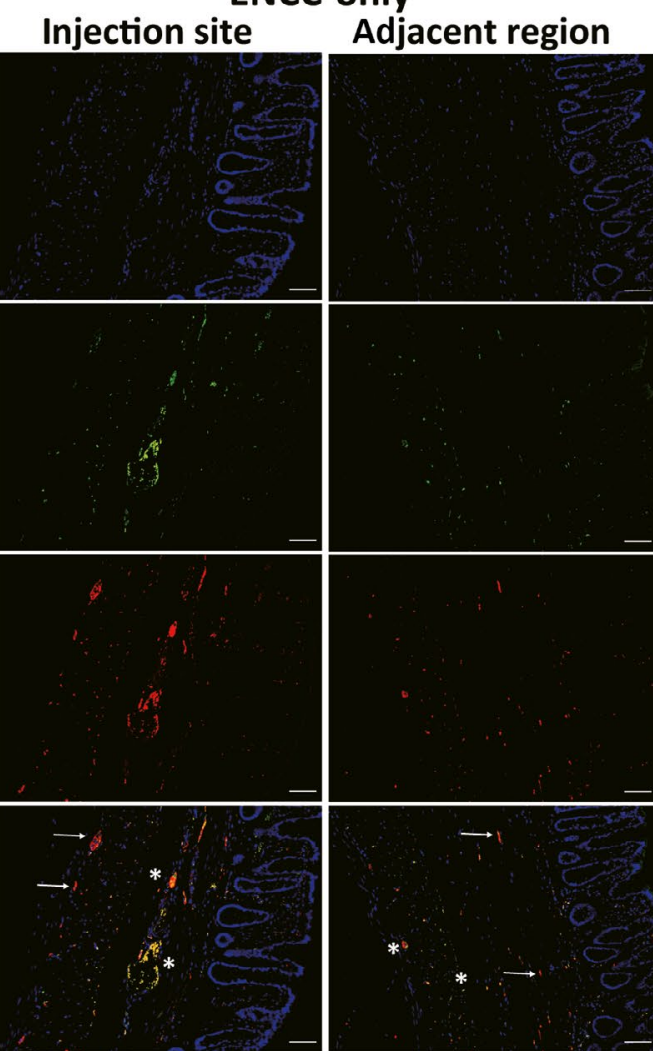

Injection site

Adjacent region

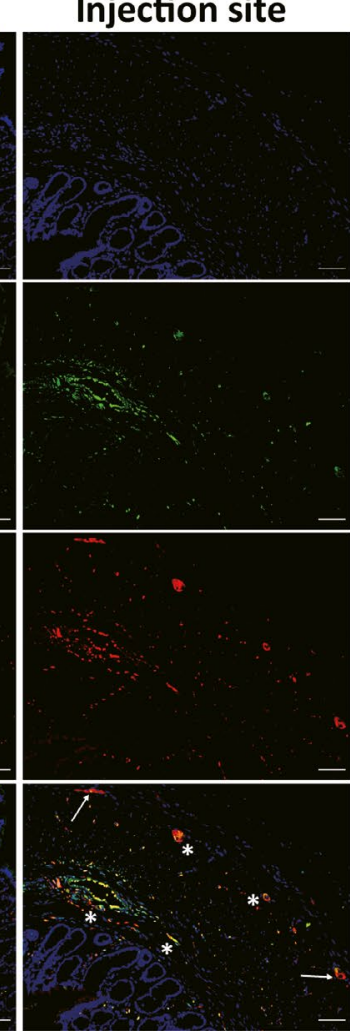

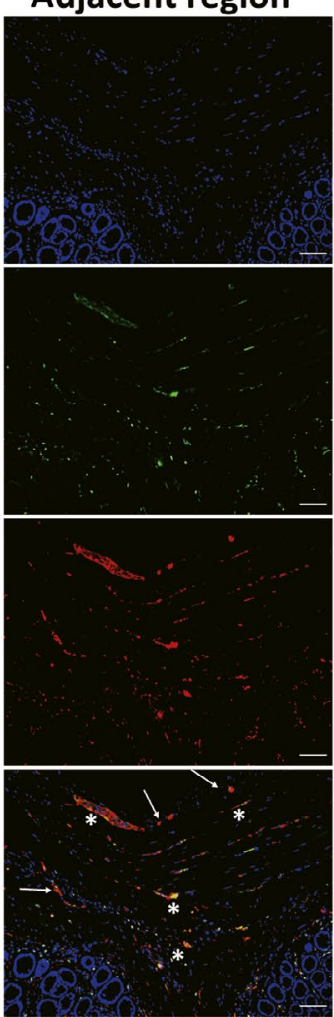


usually heterogeneous with variety of neurogenic fate potential. ${ }^{39}$ Therefore, it is still challenged to develop fully functioning ENS cells from human pluripotent stem cells, and a standard necessitated to evaluate between different protocols and derived ENS cells.

Another feasible option is to treat ENCCs with compounds during transplantation and homing. For example, serotonin and $5-\mathrm{HT}_{4}$ receptor agonists have been shown to increase the number of grafted ENCCs, leading to better transplantation outcomes. ${ }^{40,41}$ Local injection of caspase-1 inhibitor was also reported to enhance cell survival after transplantation into the gastric wall. ${ }^{42}$ Pepstatin A was reported as a candidate therapeutic drug using on human pluripotent stem cell-based platform, presenting a first example of celland drug-based strategies for the treatment of HSCR. ${ }^{43}$ In our study, we focused on the inhibition of the RhoA/ROCK pathway during ENCC-based transplantation and we showed for the first time that the ROCK inhibitor Y-27632 had a positive influence on ENCCs in vivo.

The RhoA/ROCK pathway consists of RhoA and downstream isomers, ROCK1, and ROCK2. It has been identified to play a role in neural crest specification, through ROCK and its downstream target non-muscle Myosin II. ${ }^{44}$ Relevant to our work, Y-27632 has been reported to promote neural crest generation and differentiation in a co-culture system with mouse embryonic fibroblasts. ${ }^{45}$ A possible mechanism of specification might go through YAP which has been implicated in neural differentiation. ${ }^{44}$ Alternatively, modulating ROCK might change Wnt, Bone morphogenetic proteins (BMP), or FGF signaling pathways that are known to control neural crest development. ${ }^{44,46}$ According to our results, ROCK inhibitors could increase ENCC viability, differentiation, and migration, while the RhoA inhibitor (CCG-1423) showed no effect. This indicates the existence of a direct effect of downstream ROCK isomers and a plausible multiple-pass regulation upon inhibition of RhoA.

Y-27632 is known to inhibit ROCK kinase activity, suppressing the downstream phosphorylation cascade of myosin light chain phosphatase (MLCP) and LIM kinase (LIMK). ${ }^{12}$ Both proteins are involved in actin cytoskeletal dynamics and are important for smooth muscle contraction. It is well known that intestinal motility relies on the interaction between the ENS and the smooth muscle. However, how exactly one influences the other is still unclear. A recent study identified abnormal levels of $\gamma$-smooth muscle actin in HSCR patients. ${ }^{47}$ Since the RhoA/ROCK pathway is known to be essential for smooth muscle contraction but also for axonal growth, it is tempting to speculate that the RhoA/ROCK pathway is the basis of this neural-muscle relationship.

ROCK isoforms are ubiquitously expressed throughout mammalian embryogenesis and in all adult tissues. ${ }^{48}$ In the large and small intestines, ROCK1 and ROCK2 are identified in both the epithelium and smooth muscle layers. ${ }^{49}$ In the submucosa, they are expressed in nerve cells and cell bodies of interstitial cells of Cajal (ICCs). ${ }^{50}$ The RhoA/ROCK pathway can be activated by various membrane receptors upon binding of extracellular cytokines, and in turn, it could influence other pathways. Therefore, in HSCR patients, Integrin, ${ }^{51}$ collagen, ${ }^{9,10}$ transforming growth factor (TGF- $\beta$ ), ${ }^{52}$ and $\mathrm{BMPs}^{53}$ are plausibly related to ROCK expression level as the regulated factors. Furthermore, ROCK expression in the aganglionic gut is essential for the following application of inhibitors to patients. Unfortunately, we could not confirm the expression due to the non-specificity of the antibodies used (data not shown). However, previous studies showed on the one hand increased ROCK1 expression in the aganglionic bowel of an $\mathrm{Ednrb}{ }^{(-/)}$rat model, ${ }^{21}$ but on the other hand reduced expression of ROCK1 in the aganglionic bowel of HSCR patients. ${ }^{50}$ The discrepancy between these findings might be explained by differences between humans and mice, but problems with the antibodies might also be an explanation of the differences observed. Another issue might be the different genetic backgrounds or different micro-environments of the ENS in the human and mouse gut. Clearly, additional studies are needed to determine the expression of ROCK in HSCR patients.

Apart from ENS, Y-27632 has been reported as potential therapeutic agents in a variety of diseases including liver fibrosis, ${ }^{54}$ acute respiratory distress syndrome, ${ }^{55}$ renal interstitial inflammation and fibrosis, long-term renal graft survival. ${ }^{56}$ Although not much has been mentioned about the drawbacks of Y-27632, the broad application pointed out that it will affect various tissues and organ systems, which should be considered for the use of Y-27632 in HSCR.

\section{5 | CONCLUSION}

Our study showed that inhibition of ROCK by Y-27632 could not only increase the growth, differentiation, and migration of ENCCs in vitro, but also improve the efficiency of ENCC transplantation via acceleration on graft ENCCs and endogenous ENS, leading to an improved outcome of the transplanted cells. We believe that further studies should investigate the role of the ROCK pathway in HSCR as well as other extracellular factors that play a role in ENS development. Our work emphasized the importance of the microenvironment and surrounding cells for optimal ENCC engraftment, proliferation, migration, and differentiation, which is extremely useful for improving cell-based therapeutic strategies for HCSR and other ENS-related disorders.

\section{ACKNOWLEDGMENTS}

This study was mainly supported by research grants from the National Natural Science Foundation of China (No. 81770513). Grants (National Natural Science Foundation of China, No. 81741096) and Erasmus Medical Center (Rotterdam, the Netherlands) were acknowledged for supporting this experiment and language as well.

\section{CONFLICT OF INTEREST}

The authors indicated no potential conflicts of interest.

\section{AUTHOR CONTRIBUTIONS}

Yuying Zhao involved in conception and design, data collection, animal experiment, data analysis, and manuscript writing; Xin Ge involved in cell culture and animal experiment; Hui Yu involved in cell 
culture and methodology; Laura E. Kuil involved in animal experiments and manuscript editing; Maria M. Alves involved in writing, review and editing the manuscript; Donghao Tian involved in immunohistochemistry staining and visualization; Qiang Huang involved in conception and discussion; Xinlin Chen involved in administrative support and technique support to investigation and software; Robert MW Hofstra involved in supervision, validation, and reviewed and edited the manuscript; Ya Gao involved in conception and design, financial support, project administration, supervision and validation, data analysis, and interpretation.

\section{DATA AVAILABILITY STATEMENT}

The data that support the findings of this study are available from the corresponding author upon reasonable request.

\section{ORCID}

Ya Gao iD https://orcid.org/0000-0002-6729-6374

\section{REFERENCES}

1. Burns AJ, Goldstein AM, Newgreen DF, et al. White paper on guidelines concerning enteric nervous system stem cell therapy for enteric neuropathies. Dev Biol. 2016;417(2):229-251.

2. Jiang Q, Liu F, Miao C, et al. RET somatic mutations are underrecognized in Hirschsprung disease. Genet Med. 2018;20(7):770-777.

3. Walters LC, Cantrell VA, Weller KP, Mosher JT, Southard-Smith EM. Genetic background impacts developmental potential of enteric neural crest-derived progenitors in the Sox10Dom model of Hirschsprung disease. Hum Mol Genet. 2010;19(22):4353-4372.

4. Furness JB. The enteric nervous system and neurogastroenterology. Nat Rev Gastroenterol Hepatol. 2012;9(5):286-294.

5. Pan WK, Zheng BJ, Gao Y, Qin H, Liu Y. Transplantation of neonatal gut neural crest progenitors reconstructs ganglionic function in benzalkonium chloride-treated homogenic rat colon. J Surg Res. 2011;167(2):e221-e230.

6. Lindley RM, Hawcutt DB, Connell MG, et al. Human and mouse enteric nervous system neurosphere transplants regulate the function of aganglionic embryonic distal colon. Gastroenterology. 2008;135(1):205-216.e6.

7. Hotta R, Stamp LA, Foong JP, et al. Transplanted progenitors generate functional enteric neurons in the postnatal colon. J Clin Invest. 2013;123(3):1182-1191.

8. Micci MA, Pasricha PJ. Neural stem cells for the treatment of disorders of the enteric nervous system: strategies and challenges. Dev Dyn. 2007;236(1):33-43.

9. Soret R, Mennetrey M, Bergeron KF, et al. A collagen VI-dependent pathogenic mechanism for Hirschsprung's disease. J Clin Invest. 2015;125(12):4483-4496.

10. Heuckeroth RO. Hirschsprung's disease, Down syndrome, and missing heritability: too much collagen slows migration. J Clin Invest. 2015;125(12):4323-4326.

11. Rusmini M, Griseri P, Lantieri F, et al. Induction of RET dependent and independent pro-inflammatory programs in human peripheral blood mononuclear cells from Hirschsprung patients. PLoS One. 2013;8(3):e59066.

12. Liu J, Gao HY, Wang XF. The role of the Rho/ROCK signaling pathway in inhibiting axonal regeneration in the central nervous system. Neural Regen Res. 2015;10(11):1892-1896.

13. Jaffe AB, Hall A. Rho GTPases: biochemistry and biology. Annu Rev Cell Dev Biol. 2005;21:247-269.

14. Fujita Y, Yamashita T. Axon growth inhibition by RhoA/ROCK in the central nervous system. Front Neurosci. 2014;22(8):338.
15. Frisca F, Crombie DE, Dottori M, Goldshmit Y, Pébay A. Rho/ ROCK pathway is essential to the expansion, differentiation, and morphological rearrangements of human neural stem/ progenitor cells induced by lysophosphatidic acid. J Lipid Res. 2013;54(5):1192-1206.

16. Li J, Ye L, Sanders AJ, Jiang WG. Repulsive guidance molecule B (RGMB) plays negative roles in breast cancer by coordinating BMP signaling. J Cell Biochem. 2012;113(7):2523-2531.

17. Borisoff JF, Chan CC, Hiebert GW, et al. Suppression of Rho-kinase activity promotes axonal growth on inhibitory CNS substrates. Mol Cell Neurosci. 2003;22(3):405-416.

18. Stewart AL, Young HM, Popoff M, Anderson RB. Effects of pharmacological inhibition of small GTPases on axon extension and migration of enteric neural crest-derived cells. Dev Biol. 2007;307(1):92-104.

19. Schill EM, Lake JI, Tusheva OA, et al. Ibuprofen slows migration and inhibits bowel colonization by enteric nervous system precursors in zebrafish, chick and mouse. Dev Biol. 2016;409(2):473-488.

20. Bayguinov O, Dwyer L, Kim H, Marklew A, Sanders KM, Koh SD. Contribution of Rho-kinase to membrane excitability of murine coIonic smooth muscle. Br J Pharmacol. 2011;163(3):638-648.

21. Akiyoshi J, leiri S, Nakatsuji T, Taguchi T. Mechanism of Rho-kinasemediated $\mathrm{Ca} 2+-$-independent contraction in aganglionic smooth muscle in a rat model of Hirschsprung's disease. Pediatr Surg Int. 2009;25(11):955-960.

22. Yu H, Zheng BJ, Pan WK, et al. Combination of exogenous cell transplantation and 5-HT(4) receptor agonism induce endogenous enteric neural crest-derived cells in a rat hypoganglionosis model. Exp Cell Res. 2017;351(1):36-42.

23. Rieske P, Azizi SA, Augelli B, Gaughan J, Krynska B. A population of human brain parenchymal cells express markers of glial, neuronal and early neural cells and differentiate into cells of neuronal and glial lineages. Eur J Neurosci. 2007;25(1):31-37.

24. Dráberová E, Del Valle L, Gordon J, et al. Class III beta-tubulin is constitutively coexpressed with glial fibrillary acidic protein and nestin in midgestational human fetal astrocytes: implications for phenotypic identity. I Neuropathol Exp Neurol. 2008;67(4):341-354.

25. Minami T, Kuwahara K, Nakagawa Y, et al. Reciprocal expression of MRTF-A and myocardin is crucial for pathological vascular remodelling in mice. EMBO J. 2012;31(23):4428-4440.

26. Watanabe K, Ueno M, Kamiya D, et al. A ROCK inhibitor permits survival of dissociated human embryonic stem cells. Nat Biotechnol. 2007;25(6):681-686.

27. Feng $Y$, Yin $Y$, Weiser $A$, et al. Discovery of substituted 4-(pyrazol-4-yl)-phenylbenzodioxane-2-carboxamides as potent and highly selective Rho kinase (ROCK-II) inhibitors. J Med Chem. 2008;51(21):6642-6645.

28. Natarajan D, Cooper J, Choudhury S, et al. Lentiviral labeling of mouse and human enteric nervous system stem cells for regenerative medicine studies. Neurogastroenterol Motil. 2014;26(10):1513-1518.

29. Yu H, Pan W, Ge X, et al. Nontoxicity of lentiviral vector infection to viability, migration, apoptosis, and differentiation of postnatal rat enteric neural crest-derived cells. NeuroReport. 2015;26(15):883-889.

30. Wagner JP, Sullins VF, Dunn JC. A novel in vivo model of permanent intestinal aganglionosis. J Surg Res. 2014;192(1):27-33.

31. Yu H, Pan W, Wang H, Gao Y. A time-limited and partially reversible model of hypoganglionosis induced by benzalkonium chloride treatment. Neurochem Res. 2016;41(5):1138-1144.

32. Metzger M, Bareiss PM, Danker T, et al. Expansion and differentiation of neural progenitors derived from the human adult enteric nervous system. Gastroenterology. 2009;137(6):2063-2073.e4.

33. Kruger GM, Mosher JT, Bixby S, Joseph N, Iwashita T, Morrison SJ. Neural crest stem cells persist in the adult gut but undergo changes 
in self-renewal, neuronal subtype potential, and factor responsiveness. Neuron. 2002;35(4):657-669.

34. Zhang D, Rollo BN, Nagy N, Stamp L, Newgreen DF. The enteric neural crest progressively loses capacity to form enteric nervous system. Dev Biol. 2019;446(1):34-42.

35. Yugawa T, Nishino K, Ohno S, et al. Noncanonical NOTCH signaling limits self-renewal of human epithelial and induced pluripotent stem cells through ROCK activation. Mol Cell Biol. 2013;33(22):4434-4447.

36. Ngan ES, Garcia-Barceló MM, Yip BH, et al. Hedgehog/Notchinduced premature gliogenesis represents a new disease mechanism for Hirschsprung disease in mice and humans. J Clin Invest. 2011;121(9):3467-3478.

37. Tseropoulos G, Moghadasi Boroujeni S, Bajpai VK, Lei P, Andreadis ST. Derivation of neural crest stem cells from human epidermal keratinocytes requires FGF-2, IGF-1, and inhibition of TGF- $\beta 1$. Bioeng Transl Med. 2018;3(3):256-264.

38. Lee G, Chambers SM, Tomishima MJ, Studer L. Derivation of neural crest cells from human pluripotent stem cells. Nat Protoc. 2010;5(4):688-701.

39. Lau ST, Li Z, Pui-Ling Lai F, et al. Activation of hedgehog signaling promotes development of mouse and human enteric neural crest cells. based on single-cell transcriptome analyses. Gastroenterology. 2019;157(6):1556-1571.e5.

40. Goto K, Kawahara I, Inada H, et al. Activation of 5-HT4 receptors facilitates neurogenesis from transplanted neural stem cells in the anastomotic ileum. J Physiol Sci. 2016;66(1):67-76.

41. Hotta R, Cheng L, Graham HK, et al. Delivery of enteric neural progenitors with 5-HT4 agonist-loaded nanoparticles and thermosensitive hydrogel enhances cell proliferation and differentiation following transplantation in vivo. Biomaterials. 2016;88:1-11.

42. Micci MA, Pattillo MT, Kahrig KM, Pasricha PJ. Caspase inhibition increases survival of neural stem cells in the gastrointestinal tract. Neurogastroenterol Motil. 2005;17(4):557-564.

43. Fattahi F, Steinbeck JA, Kriks S, et al. Deriving human ENS lineages for cell therapy and drug discovery in Hirschsprung disease. Nature. 2016;531(7592):105-109.

44. Kim K, Ossipova O, Sokol SY. Neural crest specification by inhibition of the ROCK/Myosin II pathway. Stem Cells. 2015;33(3):674-685.

45. Hotta R, Pepdjonovic L, Anderson RB, et al. Small-molecule induction of neural crest-like cells derived from human neural progenitors. Stem Cells. 2009;27(12):2896-2905.

46. Huang X, Saint-Jeannet JP. Induction of the neural crest and the opportunities of life on the edge. Dev Biol. 2004;275(1):1-11.

47. Moore SW, Maluleke T, EI Hosny AA. Is Hirschsprung disease a purely neurological condition? A study of the Actin G2 smooth muscle gene in Hirschsprung disease. J Pediatr Surg. 2019;54(10):2028-2031.

48. Koch JC, Tatenhorst L, Roser AE, Saal KA, Tönges L, Lingor P. ROCK inhibition in models of neurodegeneration and its potential for clinical translation. Pharmacol Ther. 2018;189:1-21.

49. lizuka M, Kimura K, Wang S, et al. Distinct distribution and localization of Rho-kinase in mouse epithelial, muscle and neural tissues. Cell Struct Funct. 2012;37(2):155-175.

50. Coyle D, O'Donnell AM, Corcionivoschi N, Gillick J, Puri P. Rhokinase expression in Hirschsprung's disease. Pediatr Surg Int. 2015;31(11):1077-1085.

51. Zhang Y, Niswander L. Phactr4: a new integrin modulator required for directional migration of enteric neural crest cells. Cell Adh Migr. 2012;6(5):419-423.

52. $\mathrm{Hagl} \mathrm{Cl}$, Rauch $\mathrm{U}$, Klotz $\mathrm{M}$, et al. The microenvironment in the Hirschsprung's disease gut supports myenteric plexus growth. Int J Colorectal Dis. 2012;27(6):817-829.

53. Wu M, Chen W, Mi J, Chen D, Wang W, Gao H. Expression analysis of BMP2, BMP5, BMP10 in human colon tissues from Hirschsprung disease patients. Int J Clin Exp Pathol. 2014;7(2):529-536.

54. Murata T, Arii S, Mori A, Imamura M. Therapeutic significance of Y-27632, a Rho-kinase inhibitor, on the established liver fibrosis. J Surg Res. 2003;114(1):64-71.

55. Köksel O, Yildirim C, Tiftik RN, et al. Rho-kinase (ROCK-1 and ROCK-2) upregulation in oleic acid-induced lung injury and its restoration by Y-27632. Eur J Pharmacol. 2005;510(1-2):135-142.

56. Liu M, Gu M, Wu Y, et al. Therapeutic effect of Y-27632 on chronic allograft nephropathy in rats. J Surg Res. 2009;157(1):e117-e127.

\section{SUPPORTING INFORMATION}

Additional supporting information may be found online in the Supporting Information section.

How to cite this article: Zhao Y, Ge X, Yu H, et al. Inhibition of ROCK signaling pathway accelerates enteric neural crest cell-based therapy after transplantation in a rat hypoganglionic model. Neurogastroenterology \& Motility. 2020;00:e13895. https://doi.org/10.1111/nmo.13895 